\title{
The IMF and star formation history of the stellar clusters in the Vela D cloud ${ }^{\star}$
}

\author{
F. Massi ${ }^{1}$, L. Testi ${ }^{1}$, and L. Vanzi ${ }^{2}$ \\ 1 INAF - Osservatorio Astrofisico di Arcetri, Largo E. Fermi 5, 50125 Firenze, Italy \\ e-mail: fmassi@arcetri.astro.it \\ 2 ESO, Alonso de Cordova 3107, Vitacura, Casilla 19001, Santiago 19, Chile
}

Received 15 July 2005 / Accepted 8 November 2005

\section{ABSTRACT}

\begin{abstract}
Aims. We present the results of a Near-Infrared deep photometric survey of a sample of six embedded star clusters in the Vela-D molecular cloud, all associated with luminous $\left(\sim 10^{3} L_{\odot}\right)$ IRAS sources. The clusters are unlikely to be older than a few $10^{6}$ yrs, since all are still associated with molecular gas.

Methods. We employed the fact that all clusters lie at the same distance and were observed with the same instrumental setting to derive their properties in a consistent way, being affected by the same instrumental and observational biases. We extracted the clusters' $K$ Luminosity Functions and developed a simple method to correct them for extinction, based on colour-magnitude diagrams. The reliability of the method has been tested by constructing synthetic clusters from theoretical tracks for pre-main sequence stars and a standard Initial Mass Function. The clusters' Initial Mass Functions have been derived from the dereddened $K$ Luminosity Functions by adopting a set of pre-main sequence evolutionary tracks and assuming coeval star formation.

Results. All clusters are small ( $\sim 100$ members) and compact (radius $\sim 0.1-0.2 \mathrm{pc}$ ); their most massive stars are intermediate-mass $\left(\sim 2-10 M_{\odot}\right)$ ones. The dereddened $K$ Luminosity Functions are likely to arise from the same distribution, suggesting that the selected clusters have quite similar Initial Mass Functions and star formation histories. The Initial Mass Functions are consistent with those derived for field stars and clusters. Adding them together we found that the "global" Initial Mass Function appears steeper at the high-mass end and exhibits a drop-off at $\sim 10 M_{\odot}$. In fact, a standard Initial Mass Function would predict a star with $M>22.5 M_{\odot}$ within one of the clusters, which is not found. Hence, either high-mass stars need larger clusters to be formed, or the Initial Mass Function of the single clusters is steeper at the high-mass end because of the physical conditions in the parental gas.
\end{abstract}

Key words. stars: formation - stars: pre-main sequence - ISM: individual objects: Vela Molecular Ridge - infrared: stars

\section{Introduction}

It is now well established by means of direct and indirect observations that most, if not all, stars are formed in groups rather than in isolation (Clarke et al. 2000; Lada \& Lada 2003). Adams \& Myers (2001) suggested that most stars form in small clusters with $\sim 10-100$ members. However, by studying a large sample of embedded clusters within $2 \mathrm{kpc}$, Lada \& Lada (2003) concluded that $90 \%$ of stars that form in embedded clusters occur in rich clusters of $>100$ members, but only $<4-7 \%$ of embedded clusters emerge from molecular clouds to become bound clusters of Pleiades age.

An important result that strongly constrains theories of massive stars and stellar clusters formation is that the stellar density of young stellar clusters seems to depend on the most

^ Based on observations collected at the European Southern Observatory, Chile. massive star in the cluster. In low-mass star forming regions, stars are usually found to form in loose groups with typical densities of a few stars per cubic parsec (Gomez et al. 1993), while high-mass stars are found in dense clusters of up to $10^{4}$ stars per cubic parsec, along with a large fraction of low-mass stars (e.g. the Orion Nebula Cluster, Hillenbrand \& Hartmann 1998). The transition between these two modes of formation should occur in the intermediate-mass regime, namely $2 \lesssim M / M_{\odot} \lesssim 15$.

In order to probe this transition, Testi et al. (1999) carried out an extensive Near-Infrared (NIR) survey searching for young clusters around optically visible intermediate-mass stars (Herbig Ae/Be stars) in the northern hemisphere. The main result of this survey is that there is a strong correlation between the spectral type of the Herbig Ae/Be stars and the membership number of the stellar groups around them. Furthermore, there is compelling evidence that the most massive stars in their sample are surrounded by denser, not only more populous, clusters. 
These findings are in qualitative agreement with models that suggest a causal relationship between the birth of a massive star and the presence of rich stellar clusters. The observed correlation and scatter, however, could also be explained in terms of random assembling clusters with membership size distribution of the form $g(N) \sim N^{-1.7}$ picking stars from a standard Initial Mass Function (IMF; Bonnell \& Clarke 1999).

As discussed in Testi et al. (2001a) there are two observational strategies that may provide additional constraints on which of the two scenarios is the most appropriate: expand the sample of optically revealed young $\mathrm{O}$ and $\mathrm{B}$ stars to increase the statistic, and search for clusters in complete samples of luminous embedded sources in giant molecular clouds. Recently, de Wit et al. (2004, 2005), following the first approach, investigated the possible origin in clusters of field O-stars; they demonstrated that the majority of the so called "field" O-stars may have originated in clusters, but could not exclude that a small fraction of these are actually born in isolation. An example of the second approach is given in Massi et al. (2000, 2003) as described in Sect. 2.

One fundamental property of clusters is their IMF; different methods to extract the IMF from photometric and/or spectroscopic data are summarized in Lada \& Lada (2003). These authors concluded that embedded young clusters exhibit strong similarities in their IMF with open clusters and field stars, although this "universal" IMF does not seem to characterize all star forming regions (Lada \& Lada 2003). Scalo (1998), reviewing all determinations of the IMF in different environments, noted that either the observational uncertainties are so large as not to allow one to draw definite conclusions on the average IMF and its variation, or the available data show strong evidence for IMF variations. This is a critical issue, since the IMF should represent the imprint on a stellar population of the physical conditions in the parental gas.

Most of the main topics concerning the formation of star clusters are then still debated and ask for large observational efforts. In this paper, we report the results of a NIR study of a sample of small clusters in the giant molecular cloud " $D$ " of the Vela Molecular Ridge. Small young embedded clusters can prove very interesting targets to address many of the illustrated points, since they 1) are associated with the intermediate-mass transition regime investigated by Testi et al. (1999), 2) are on the border between the different sizes envisaged by Adams \& Myers (2001) and Lada \& Lada (2003) as major star contributors, and 3) do not host stars massive enough to heavily affect the parental environment, maintaining a stronger link with it. All imaged clusters are young and are associated with the same molecular cloud. On the one hand, this allows one to circumvent many effects due to observational biases in comparing the results. On the other hand, local differences in the global IMF emerging from the molecular cloud can be searched for and investigated. Furthermore, our data considerably improve on previous NIR photometry of the same regions, both in sensitivity and in field of view. The Paper layout is as follows: in Sect. 2, the main features of the star forming region and the selected sample are summarized. Observations and data reduction are described in Sect. 3. The main results are reported in Sect. 4 and discussed in Sect. 5. Our conclusions are listed in Sect. 6.
Table 1. Selected fields. The last column lists the designation adopted by Liseau et al. (1992).

\begin{tabular}{cccccccc}
\hline \hline IRAS source & \multicolumn{3}{c}{$\alpha(1950.0)$} & \multicolumn{3}{c}{$\delta(1950.0)$} & \\
& $\mathrm{h}$ & $\mathrm{m}$ & $\mathrm{s}$ & $\circ$ &, & \\
& & & & \\
\hline IRAS 08438-4340 & 08 & 43 & 50.2 & -43 & 40 & 02 & IRS 16 \\
IRAS 08448-4343 & 08 & 44 & 49.4 & -43 & 43 & 27 & IRS 17 \\
IRAS 08470-4243 & 08 & 47 & 00.0 & -42 & 43 & 12 & IRS 18 \\
IRAS 08470-4321 & 08 & 47 & 01.3 & -43 & 21 & 15 & IRS 19 \\
IRAS 08476-4306 & 08 & 47 & 39.4 & -43 & 06 & 01 & IRS 20 \\
IRAS 08477-4359 & 08 & 47 & 47.1 & -43 & 59 & 34 & IRS 21 \\
\hline
\end{tabular}

\section{The Vela-D luminous IRAS sources}

The Vela Molecular Ridge (VMR) is a giant molecular cloud complex located in the outer Galaxy $\left(257^{\circ}<l<274^{\circ}\right)$ within the galactic plane $\left(-5^{\circ}<b<5^{\circ}\right)$. It was first mapped in the $\mathrm{CO}(1-0)$ transition (with low resolution) by Murphy \& May (1991), who divided the emission area into 4 main regions (named A, B, C and D) corresponding to local maxima. The kinematical distance is $1-2 \mathrm{kpc}$.

Liseau et al. (1992) and Lorenzetti et al. (1993) studied the star formation in the VMR by selecting the IRAS point sources with red colours and combining their Far-Infrared (FIR) fluxes with NIR and 1.3-mm photometry. They list a sample of IRAS sources which, based on the SEDs from NIR to FIR wavelengths and the radial velocity of the associated molecular gas, may be considered as intermediate-mass $\left(M \sim 2-10 M_{\odot}\right)$ analogues of Class I sources belonging to the VMR. Liseau et al. (1992) also discuss the issue of distance, concluding that clouds A, C and D are at $d=700 \pm 200$ pc, whereas cloud B is further out ( $2 \mathrm{kpc})$. Massi et al. (2000) analyzed JHK images of the fields towards 12 sources of the sample, namely those associated with cloud $\mathrm{D}$, whose bolometric luminosities range from $\sim 10^{2}$ to $\sim 6 \times 10^{3} L_{\odot}$ (Massi et al. 1999). These authors found young embedded star clusters towards the IRAS sources with $L_{\text {bol }} \gtrsim 10^{3} L_{\odot}$. Five of these embedded clusters have been selected for the present work. In addition, we have included IRAS 08438-4340 (IRS 16, as designated by Liseau et al. 1992), a FIR source associated with both an embedded star cluster (Massi et al. 2003) and an HII region, close (in projection) to the other ones. The six fields are listed in Table 1 along with their equatorial coordinates and the nomenclature used by Liseau et al. (1992).

\section{Observations and data reduction}

The six fields towards the selected IRAS sources in the Vela D molecular cloud were observed at the ESO-NTT telescope on February 18, March 5, March 20 and September 25, 2000. Near-Infrared images through the standard $J, H$, and $K_{\mathrm{s}}$ broad band filters were acquired using the SofI instrument, with a plate scale of $\sim 0.282^{\prime \prime} /$ pixel and an instantaneous field of view of $\sim 5^{\prime}$. For each target we collected a set of dithered exposures for a total integration time of 15 minutes in each filter. The raw images were crosstalk corrected, flat fielded, sky subtracted, 


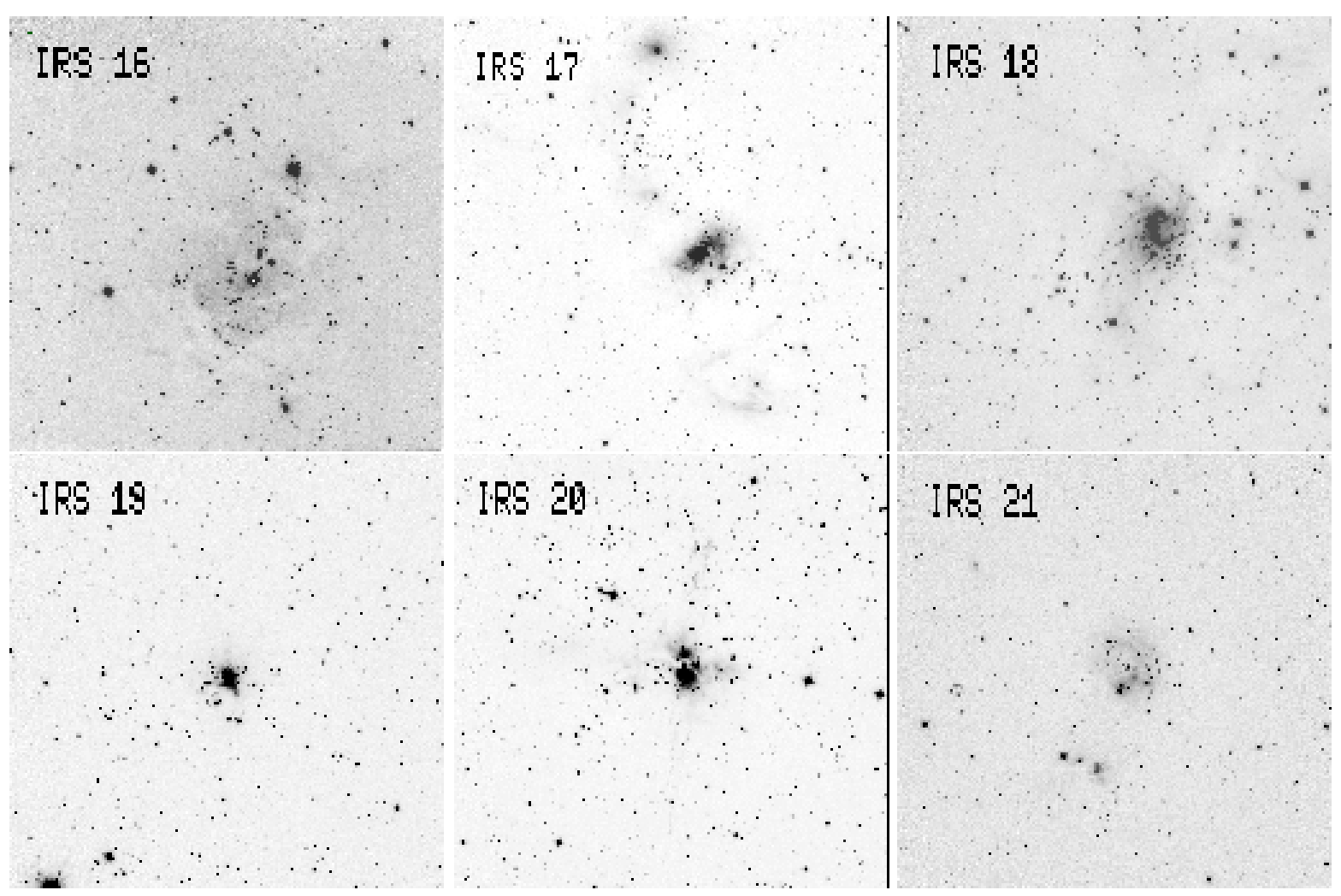

Fig. 1. NTT/SofI Near-Infrared images (through the $K_{\mathrm{s}}$ band) of the fields centred on the selected luminous IRAS sources associated with the Vela D molecular cloud.

aligned and mosaiced using the special procedures developed for SofI and standard routines within the IRAF package.

The final image quality of our images was $\sim 0.85^{\prime \prime}$ for all but the field surrounding IRS 16 which was observed under slightly worst seeing conditions (1.1"). Photometric calibration was ensured by observations of a number of standard stars from the list of Persson et al. (1998). Source detection and aperture photometry were performed using standard routines within IRAF.

We compared our photometry with that of Massi et al. (1999) and Massi et al. (2003) to check for consistency. The latter was obtained from Irac $2 \mathrm{JHK}$ images. The mean differences (in the sense Irac 2 minus SofI) are: $\sim 0$ to -0.07 mag (excepted IRS 19 and IRS 21 , with -0.34 and $-0.16 \mathrm{mag}$ ) in $K,-0.06$ to $0.01 \mathrm{mag}$ (excepted IRS 17 and IRS 21, with 0.3 and $-0.2 \mathrm{mag}$ ) in $H$ and -0.1 to $0.08 \mathrm{mag}$ (excepted IRS 17 and IRS 16, with 0.26 and $-0.16 \mathrm{mag}$ ) in $J$. Part of the largest mean differences may be attributed to problems with the aperture correction of some Irac2 data sets, as already noted by those authors (Massi et al. 2000; see also their Fig. 1 for IRS 17 and IRS 19). The scatter of the differences around the average values are 0.11 to 0.26 mag in $K, 0.12$ to 0.19 mag (excepted IRS 16 and IRS 17, with 0.34 and $0.33 \mathrm{mag}$ ) in $H$ and 0.21 to $0.25 \mathrm{mag}$ (excepted IRS 16 and IRS 17, with 0.36 and $0.30 \mathrm{mag}$ ) in $J$. We found also a colour term in $K$ of the order of $\sim-0.2 \times(H-K)$, certainly due to the different $K$ (Irac2) and $K_{\mathrm{S}}$ (SofI) filters, that may account for another part of average and r.m.s. differences. In $H$ and $J$ the colour term, if any, is much smaller: $\sim 0.04-0.07 \times(J-H)$. We believe that the relatively large scatter in the magnitude differences may be explained by the differences in PSF sampling and sky estimate between the data sets and the intrinsic variability of young stars. Anyway, both data sets appear to be mutually consistent.

\section{Results}

In Fig. 1 we show the NTT/SofI $K_{\mathrm{S}}$ images of the six surveyed fields. True-colour images (combining the images in all three bands) are shown in Testi et al. (2001b; see their Fig. 1). From those images it is immediately clear that we detected groups of very red sources in every fields.

\subsection{Mass sensitivity}

We estimate a completeness magnitude $K_{\mathrm{s}}^{\mathrm{c}} \sim 18$ for all fields, excepted IRS 21 for which $K_{\mathrm{s}}^{\mathrm{c}} \sim 17$ (see Fig. 2 in Testi et al. 2001b). Assuming a distance modulus of $9.22 \mathrm{mag}$, this allows an estimate of the minimum mass, as a function of age and reddening, for a star whose probability to be found is still almost 1 throughout each $K_{\mathrm{s}}$ frame. Using the evolutionary tracks for pms stars given by Palla \& Stahler (1999), the mass completeness limit is $M_{\text {compl }} \sim 0.1 M_{\odot}$ for $A_{\mathrm{V}}=30 \mathrm{mag}, K_{\mathrm{s}}^{\mathrm{c}} \sim 17$ and an age of $10^{6} \mathrm{yrs}$ and increases to $\sim 0.4 M_{\odot}$ for an age of $10^{7} \mathrm{yrs}$. At this age, for $A_{\mathrm{V}}=20 \mathrm{mag}$ or $K_{\mathrm{s}}^{\mathrm{c}}=18, M_{\text {compl }}$ is still as low as $0.2 M_{\odot}$. We find the same using the evolutionary tracks from D'Antona \& Mazzitelli (1994) for $10^{6}$ yrs old stars, but $M_{\text {compl }}$ ranges from $\sim 0.5 M_{\odot}\left(A_{\mathrm{V}}=30 \mathrm{mag}\right)$ to $\sim 0.3 M_{\odot}$ $\left(A_{\mathrm{V}}=20 \mathrm{mag}\right.$ or $\left.K_{\mathrm{s}}^{\mathrm{c}}=18\right)$ for $10^{7} \mathrm{yrs}$ old stars. From Baraffe et al. (1998) the estimated $M_{\text {compl }}$ is $\sim 0.15 M_{\odot}$ for $2 \times 10^{6} \mathrm{yrs}$ 


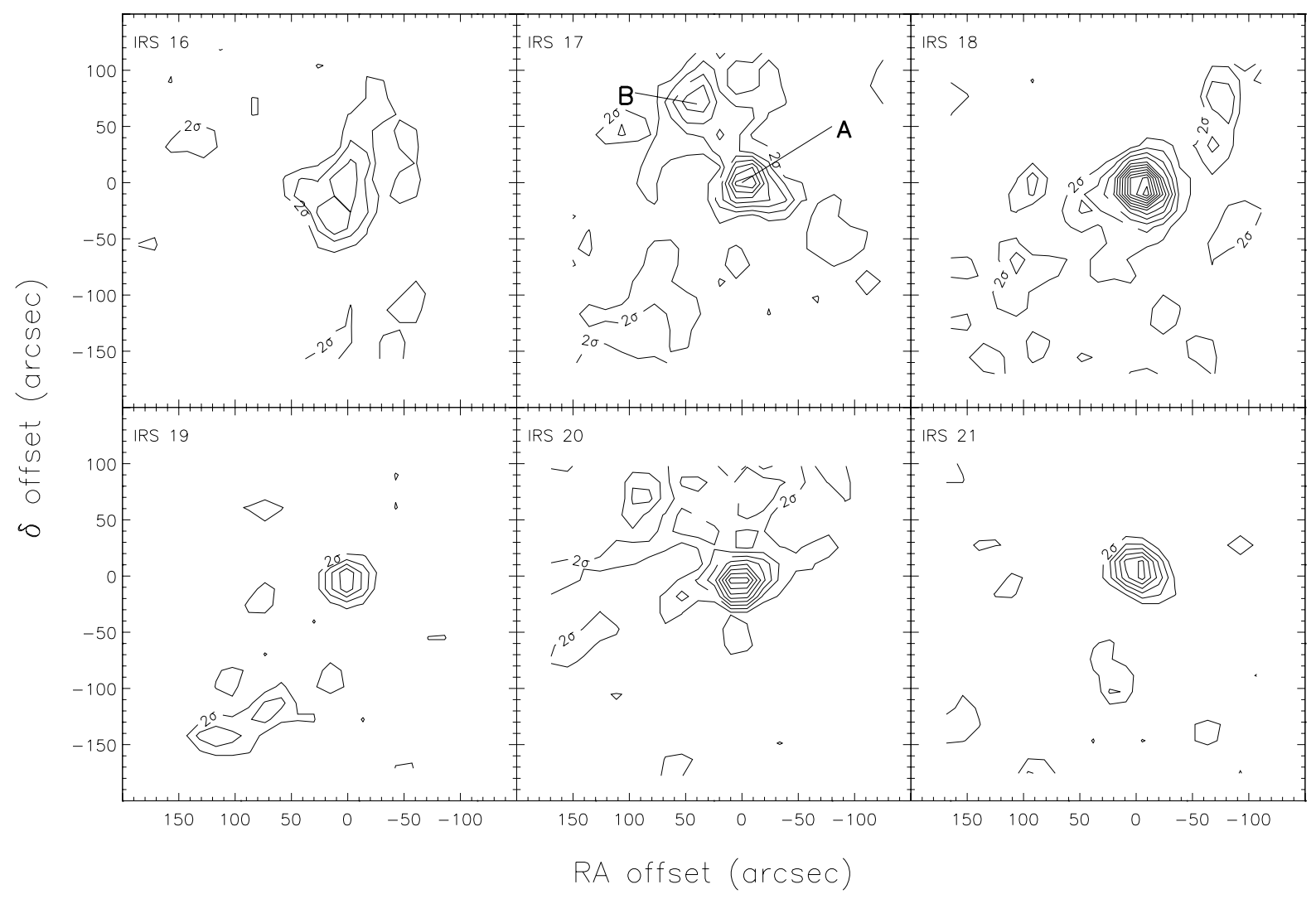

Fig. 2. Star surface density $\left(\operatorname{arcmin}^{-2}\right)$ from the $K_{\mathrm{s}}$ images of the 6 fields. Contours are in steps of $\sim 2 \sigma\left(20 \operatorname{arcmin}^{-2}\right)$ from $\sim 2 \sigma$ above the mean surface density of field stars. Towards IRS 17, two nearby subclusters (A and B) are clearly present.

old stars and $\gtrsim 0.6 M_{\odot}$ for $10^{7}$ yrs old stars. It is clear that our images probe the clusters stellar population down to the hydrogen burning limit for ages $<10^{6} \mathrm{yrs}$ and as low as $0.5 M_{\odot}$ for an older population $\left(10^{7} \mathrm{yrs}\right)$; they are probably deep enough to probe the brown dwarf regime for ages $\sim 10^{5}$ yrs. However, we caution against the decrease in completeness magnitude that is likely to occur towards the clusters' centres because of crowdedness, diffuse emission and, sometimes, the PSF wings of the brightest stars.

\subsection{Clustering}

Although the star clusters are clearly visible in the images of Fig. 1, we employ the same graphical representation as used by Massi et al. (2000) in order to better evidence their presence. Hence, we counted all $K_{\mathrm{s}}$ sources up to $K_{\mathrm{s}}=18$ in $\sim 30^{\prime \prime} \times 30^{\prime \prime}$ squares ("bins"), at offsets of $\sim 15^{\prime \prime}$ both in right ascension and in declination. Following Massi et al. (2000), we estimated the field star density (the "sky") and its $1 \sigma$ fluctuation by gathering all the bins from the 6 fields and constructing a global histogram of the number of bins with given counts. The rising part of the histogram is well fitted by a Poissonian curve with a mean of 7.5 counts per bin (32 stars $\operatorname{arcmin}^{-2}$ ), so we assume that this represents the statistic of field stars. The actual data distribution exceeds the Poissonian curve in the wing and, as noted by Massi et al. (2000), this indicates that the clustering is not due to sky fluctuations. The Poissonian fit yields a $\sigma \sim 12 \operatorname{stars} \operatorname{arcmin}^{-2}$.
Since our imaged fields are large enough, we constructed a histogram for each frame and by the same technique we obtained sky means ranging from 26 to 34 stars $\operatorname{arcmin}^{-2}$ (and $\sigma \sim 10-12$ stars $\operatorname{arcmin}^{-2}$ ). These values compare quite well with those found by Massi et al. (2000), i.e., a sky mean $\sim 20$ stars $\operatorname{arcmin}^{-2}$ and a $\sigma \sim 12 \operatorname{stars} \operatorname{arcmin}^{-2}$, considering that their photometry has a limiting magnitude $K \sim 18$ and, hence, a lower completeness magnitude than ours. Figure 2 shows the contour maps of surface star density from the $K_{\mathrm{s}}$ counting; contours start at 50 stars $\operatorname{arcmin}^{-2}$ (roughly the sky

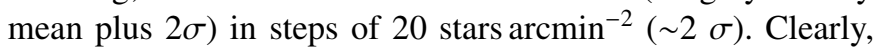
all clusters have maximum densities well above the sky mean plus $3 \sigma$. This confirms that the structures found by Massi et al. (2000) on smaller field-size images are actually young star clusters. The clusters are compact enough to fit the $2^{\prime} \times 2^{\prime}$ f.o.v. of the images used by those authors. However, the larger f.o.v. allowed by SofI and our much deeper imaging evidence some substructuring, such as the small source grouping (which we name "B") north-east of the cluster found by Massi et al. towards IRS 17 (which we name "A").

We determined the radial distribution of surface star density as described in Testi et al. (1999), shown in Fig. 3. Counting is performed on the sources with at least a detection in $K_{\mathrm{s}}$ and up to $K_{\mathrm{s}}=17$, which is the smaller completeness magnitude we obtained throughout the fields. A significant density increase towards $r=0 \mathrm{pc}$ is noticeable in all the six fields. This also allows an estimate of the surface density due to field stars by averaging the radial density in the wing of the distribution. 


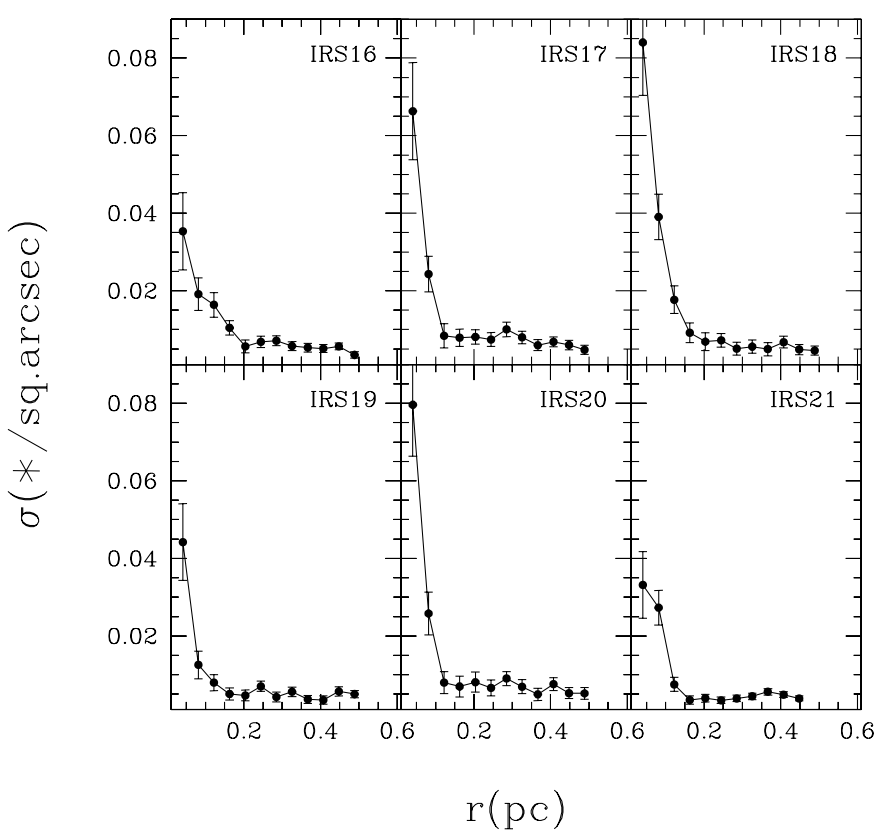

Fig. 3. Radial distribution of star surface density $\left(\operatorname{arcsec}^{-2}\right)$ from the $K_{\mathrm{s}}$ images of the 6 fields.

Table 2. Clusters' properties. The diameter is listed in Col. 2, the peak surface stellar density in Col. 3, the richness indicator (see text) in Col. 4 and the volume stellar density in Col. 5 .

\begin{tabular}{ccccc}
\hline \hline $\begin{array}{c}\text { Field } \\
\text { name }\end{array}$ & Size & $\begin{array}{c}\text { Max. surf. } \\
\text { density } \\
(\mathrm{pc})\end{array}$ & $I_{\mathrm{c}}$ & $\begin{array}{c}\text { Vol. } \\
\text { density } \\
\left(\mathrm{pc}^{-3}\right)\end{array}$ \\
\hline IRS 16 & 0.26 & 100 & $71 \pm 11$ & 1830 \\
IRS 17A & 0.21 & 180 & $50 \pm 8$ & 1289 \\
IRS 17B & 0.22 & 120 & & \\
IRS 18 & 0.27 & 270 & $118 \pm 13$ & 3042 \\
IRS 19 & 0.13 & 120 & $35 \pm 8$ & 902 \\
IRS 20 & 0.21 & 190 & $59 \pm 8$ & 1521 \\
IRS 21 & 0.16 & 150 & $52 \pm 8$ & 1340 \\
\hline
\end{tabular}

Obtained values range between 14 and $25 \mathrm{stars} / \mathrm{arcmin}^{2}$ and are consistent with the one of $32 \mathrm{stars} / \mathrm{arcmin}^{2}$ we infer above, considering the latter is obtained for sources up to $K_{\mathrm{s}}=18$. The richness indicator $I_{C}$ (Testi et al. 1999) is derived by integrating the radial stellar surface density, once subtracted the background-foreground contribution as estimated from the wing of the distribution. This is listed in Table 2 along with the statistical error due to the sky determination and the Poissonian fluctuations in the surface density of the cluster members. Figure 3 shows that all clusters have a rather similar size and allows to estimate a radius $\sim 0.1-0.2 \mathrm{pc}(\sim 0.5-1 \mathrm{arcmin})$ at the assumed distance (see Table 2).

\subsection{Cluster star population}

The nature of the star population associated with the embedded clusters can be studied through colour-colour ( $J-H$ vs. $\left.H-K_{\mathrm{s}}\right)$ and colour-magnitude $\left(K_{\mathrm{s}}\right.$ vs. $\left.H-K_{\mathrm{s}}\right)$ diagrams. Exploiting our field of view, we compare the colours of sources within 2 arcmin of the cluster centre with those of sources outside. This distance is slightly more than twice the clusters' radius; in fact, we expect the radial profiles to exhibit a lower density tail far from the centre, so we chose a value of 2 arcmin in order to account for this. Radial density profiles on a much larger scale than our images, obtained from 2MASS data, actually confirm the presence of a tail up to at least twice the clusters' radius. Hence, the sky area outside this distance is less contaminated by clusters' members and more representative of the population of field stars. Figure 4 shows the colour-colour diagrams (CCDs) for objects within the chosen radius, whereas Fig. 5 shows the same but for objects outside, both up to $K_{\mathrm{s}}=18$. The locus of main sequence (from Koornneef 1983) is drawn as a solid line and the dashed lines are parallel to the reddening vector (with crosses at intervals of $A_{\mathrm{V}}=10 \mathrm{mag}$ ) according to the extinction law given by Rieke \& Lebofsky (1985). The NIR sources $>2$ arcmin far from the clusters' centres are essentially reddened stars, up to $A_{\mathrm{V}} \sim 10-15 \mathrm{mag}$; the dispersion around the main sequence and its reddening band denotes a large fraction of faint objects that may represent background field stars. There are few NIR sources with an intrinsic colour excess. Conversely, within the chosen radius, a different stellar population emerges from the previous one; the objects now spread over a larger extinction range, up to $A_{\mathrm{V}} \sim 20-30 \mathrm{mag}$, there is less dispersion around the main sequence and the fraction of NIR sources with an intrinsic colour excess is much larger. This indicates that the peak extinction is greater towards the clusters' centres (hence, most background stars are reddened out) and that a population of very young stars is superposed to that of field stars which characterizes the outer edge of the imaged fields. Including only sources with $K_{\mathrm{s}} \leq 15$ further limits the dispersion around the main sequence and reddening bands, but does not alter the spread along the extinction vector and the high fraction of NIR objects with intrinsic colour excess near to the clusters' centre. Also, note that the observed reddening follows quite well the drawn loci, confirming that the extinction law towards the 6 fields is consistent with the standard one as given by Rieke \& Lebofsky (1985).

All the previous findings can be checked using colourmagnitude diagrams (CMDs). These are presented in Fig. 6 and Fig. 7 for all fields. The solid line is the locus of Zero Age Main Sequence (ZAMS) at the distance of Vela (700 pc) using the absolute magnitudes given by Allen (1976) and the colours given by Koornneef (1983). The location of B0, A0, K0 and M5 stars are labelled. The arrow is for a reddening $A_{\mathrm{V}}=20$ mag according to Rieke \& Lebofsky (1985). It can be seen that the sources far from the clusters' centres (see Fig. 7) are on average closer to the ZAMS (i.e., less extincted) and a large fraction of them is fainter than $K_{\mathrm{s}} \sim 16$ (i.e., they are mostly background field stars as noted above). Actually, the fraction of sources (up to $K_{\mathrm{s}}=18$ ) with $K_{\mathrm{s}}<16$ within/outside 2 arcmin from the centres ranges from $0.46 / 0.33$ (IRS 16) to 0.36/0.29 (IRS 20). Furthermore, the sources within 2 arcmin from the clusters' centres are in a larger fraction far from the ZAMS, because of both their greater extinction and their intrinsic colour excess: the fraction of sources (up to $K_{\mathrm{s}}=18$ ) with $H-K_{\mathrm{s}}>1.5 \mathrm{mag}$ within/outside 2 arcmin from the centres 


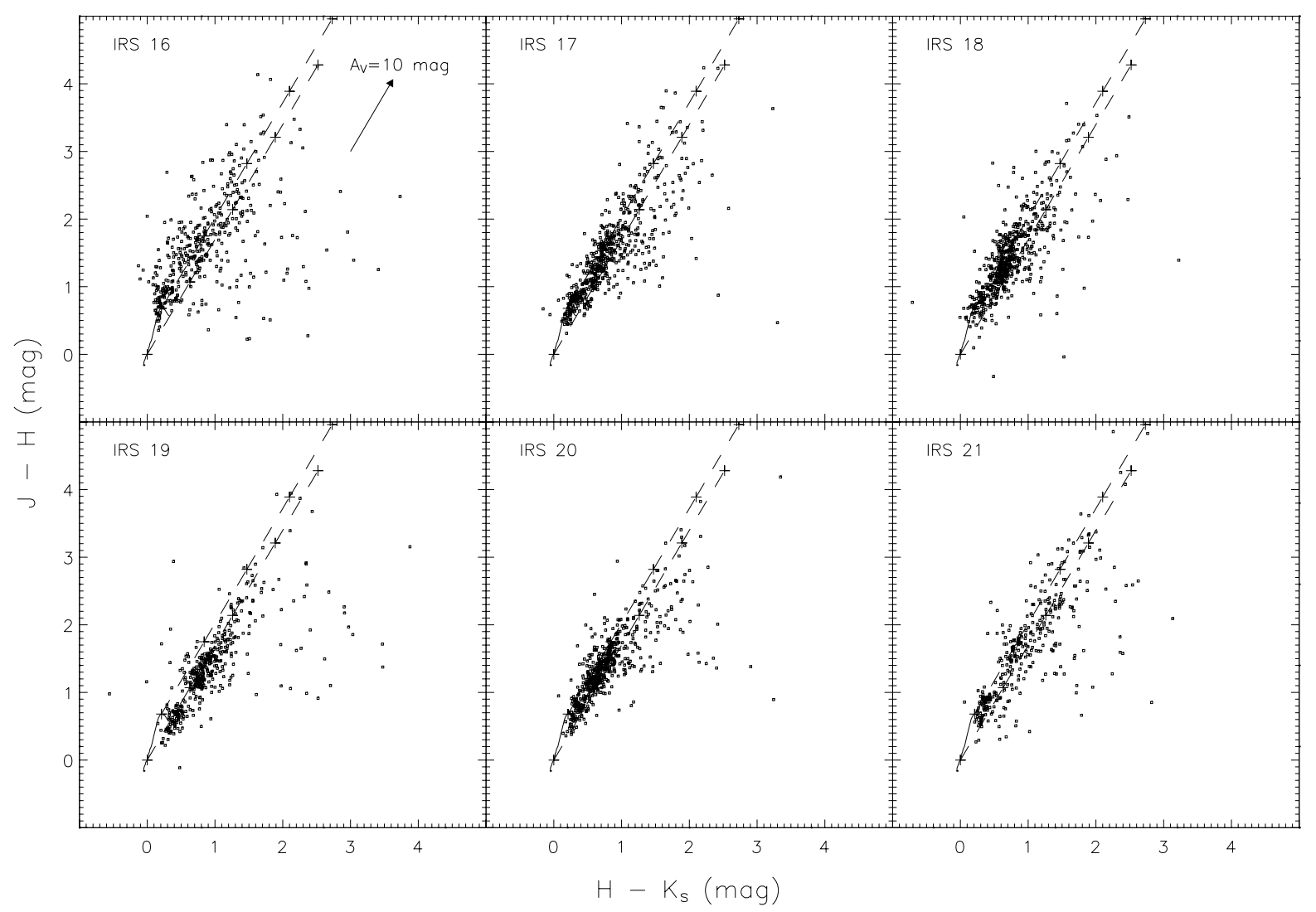

Fig. 4. Colour-colour diagrams (i.e., $J-H$ vs. $H-K_{\mathrm{s}}$ ) of the 6 fields, for objects lying within 2 arcmin (i.e., roughly twice the clusters' radius) from the clusters' centres and up to $K_{\mathrm{s}}=18$. The solid line marks the main sequence (Koornneef 1983) whereas the dashed ones indicate the reddening law with crosses at intervals of $A_{\mathrm{V}}=10$ mag (Rieke \& Lebofsky 1985). For the sake of clarity, an arrow corresponding to an extinction $A_{\mathrm{V}}=10 \mathrm{mag}$ has been also drawn, in the upper left panel.

ranges from $0.18 / 0.03$ (IRS 16) to $0.07 / 0.007$ (IRS 18). This confirms that the clusters' stellar population is represented by very young, embedded stars, mostly located within 2 arcmin (or even less) from the centres and that it is indeed different from the one of field stars.

\subsection{The clusters' K Luminosity Functions}

The $K$ Luminosity Function (KLF) of an embedded cluster is a simple tool to probe its Initial Mass Function (IMF) and age. As discussed, e.g., by Zinnecker et al. (1993), the KLF depends not only on the underlying IMF, but also on the mass-luminosity relation. For pms stars the mass-luminosity relation is a function of age. Hence, given the IMF, a cluster's KLF evolves with time and also changes according to the star formation history of the cluster. However, Muench et al. (2000) show that the KLF of a young cluster is much more sensitive to variations in its underlying IMF than to the star formation history or the massluminosity relation. Also, a cluster KLF shifts to fainter magnitudes and widen systematically with age. This should allow to put some constraints on the age of a young cluster, as well.

The KLFs obtained from our $K_{\mathrm{s}}$ photometry are shown in Fig. 2 of Testi et al. (2001b). For each field, two KLFs are determined: one using sources close to the cluster centre and one using sources at the image edge. Clearly, the two sets of distributions differ in shape, confirming that the clusters host a star population different from the field one, as inferred in Sect. 4.3 on the basis of CCDs and CMDs. In particular, the outer KLFs increase steadily and peak at higher magnitudes than the "central" KLFs. This maximum reflects the completeness limit at $K_{\mathrm{s}}$. Since at the edges of our images the number of cluster members is small with respect to field stars, we chose to construct "control" KLFs using the photometry obtained in the outer areas themselves of our frames. These should better characterize the contribution of field stars to the raw KLFs than those obtained using relatively distant control regions. We adopted a radius of $2^{\prime}$ from the cluster centre to define the border between "centre" and "edge". The cluster's centre is assumed to coincide with the NIR counterparts of the IRAS sources identified by Massi et al. (1999). The reason for our choice of the radius is explained in Sect. 4.3; actually a larger radius would assure a much less contamination, but would also decrease the outer area available for estimating the control KLF.

Thus, we constructed the two KLFs per field by counting the $K_{\mathrm{s}}$ sources in bins of $0.5 \mathrm{mag}$, within and outside the selected radius. After normalizing the outer KLFs to the same area of the central ones, the clusters' KLFs were finally obtained by subtracting the former from the latter in each field. Yet, in order to derive the clusters' IMFs from their KLFs, source extinction and NIR excess have to be accounted for. Extinction causes a twofold problem. First, even with so nearby 


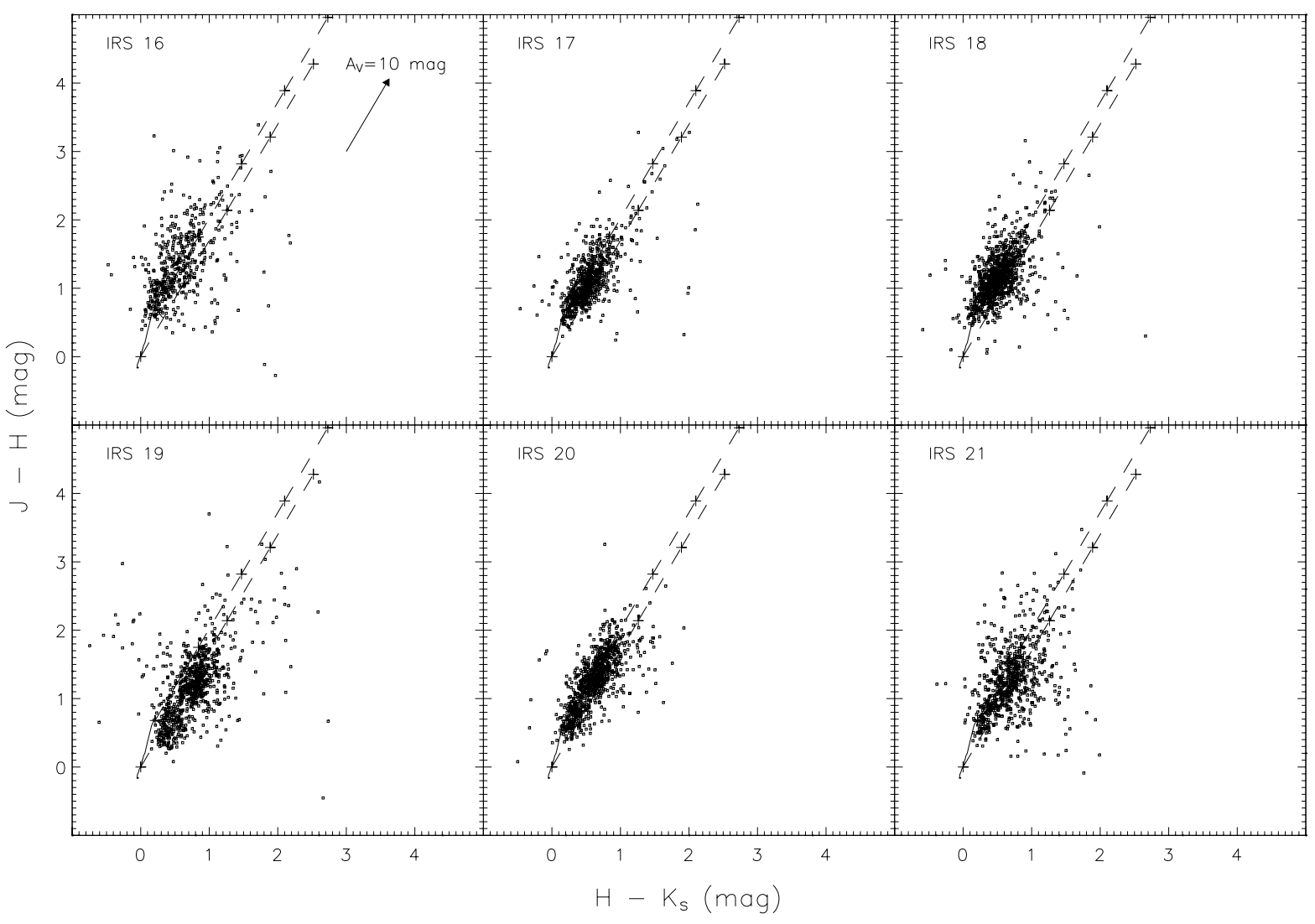

Fig. 5. Same as Fig 4, but for objects further than 2 arcmin from the clusters' centres.

control fields the sources at the centre and those at the edge of each image are reddened in different ways, as evidenced by the CCDs. Furthermore, the single clusters' members are differentially reddened and, because the span in $A_{\mathrm{V}}$ may be more than 10 mag (i.e., more than $1 \mathrm{mag}$ in $A_{K}$ ), this cannot be neglected even binning the KLFs at a large interval. Thus, we tested two methods in order to account for the effect of extinction on the KLFs: modelling the reddening of theoretical KLFs, and trying to "deredden" the observed KLFs. We found the latter method more satisfactory and we discuss it in the following.

\subsection{Modelling K-Luminosity functions}

We performed several tests with synthetic KLFs, which are briefly discussed in the following sections. To do that, we developed a Montecarlo procedure to construct theoretical KLFs by assuming an underlying IMF, an age and a star formation history. The adopted mass-luminosity relation has been derived from the evolutionary tracks of Palla \& Stahler (1999). The procedure makes an estimate of the number of cluster members on the basis of the bolometric luminosities that is selected, using an empirical relation between $I_{\mathrm{c}}$ and $L_{\mathrm{bol}}$ derived from the data of Testi et al. $(1997,1998)$. The NIR colour excess has been modelled as in Hillenbrand \& Carpenter (2000) and it is also possible to add to the NIR fluxes a random reddening (according to a Gaussian statistic) superposed to a constant value.

\subsection{Dereddening the observed K Luminosity Functions}

There are indications that the surveyed clusters are at most a few $10^{6}$ yrs old: their compactness, the high spread in extinction displayed by their members and a significant fraction of sources exhibiting a NIR infrared excess in the CCDs (see Fig. 4). According to Lada \& Lada (2003), the embedded phase of cluster evolution lasts 2-3 Myrs and clusters older than 5 Myrs are rarely associated with molecular gas. All our observed clusters are associated with molecular gas (see Massi et al. 2000, 2003, 2005).

Their youth was also confirmed through a preliminary modelization, constructing reddened theoretical KLFs based on a rough estimates of the extinction distribution in the clusters according to the CCDs. The comparison with the observed cluster KLFs indicates that the clusters' age roughly ranges between $\sim 10^{6}$ and $\sim 10^{7}$ yrs. This suggested us a simple method in order to deredden the observed KLF, which is illustrated in Fig. 8. Figure 8 a details a CMD ( $K$ vs. $H-K$ ) including the ZAMS locus (solid line) and three isochrones from the evolutionary pms tracks of Palla \& Stahler (1999; dashed lines), all for a distance of $700 \mathrm{pc}$. Clearly, pms stars from $10^{5}$ years old to the ZAMS span quite a limited range in colour (0.2-0.4 mag) and, at the same time, the slope of the reddening law is small. This means that the stars may be dereddened on a CMD simply by projecting them back along the reddening vector onto a mean locus (thick line in Fig. 8) chosen to be located between the isochrones. The error is small; e.g., for pms stars older 


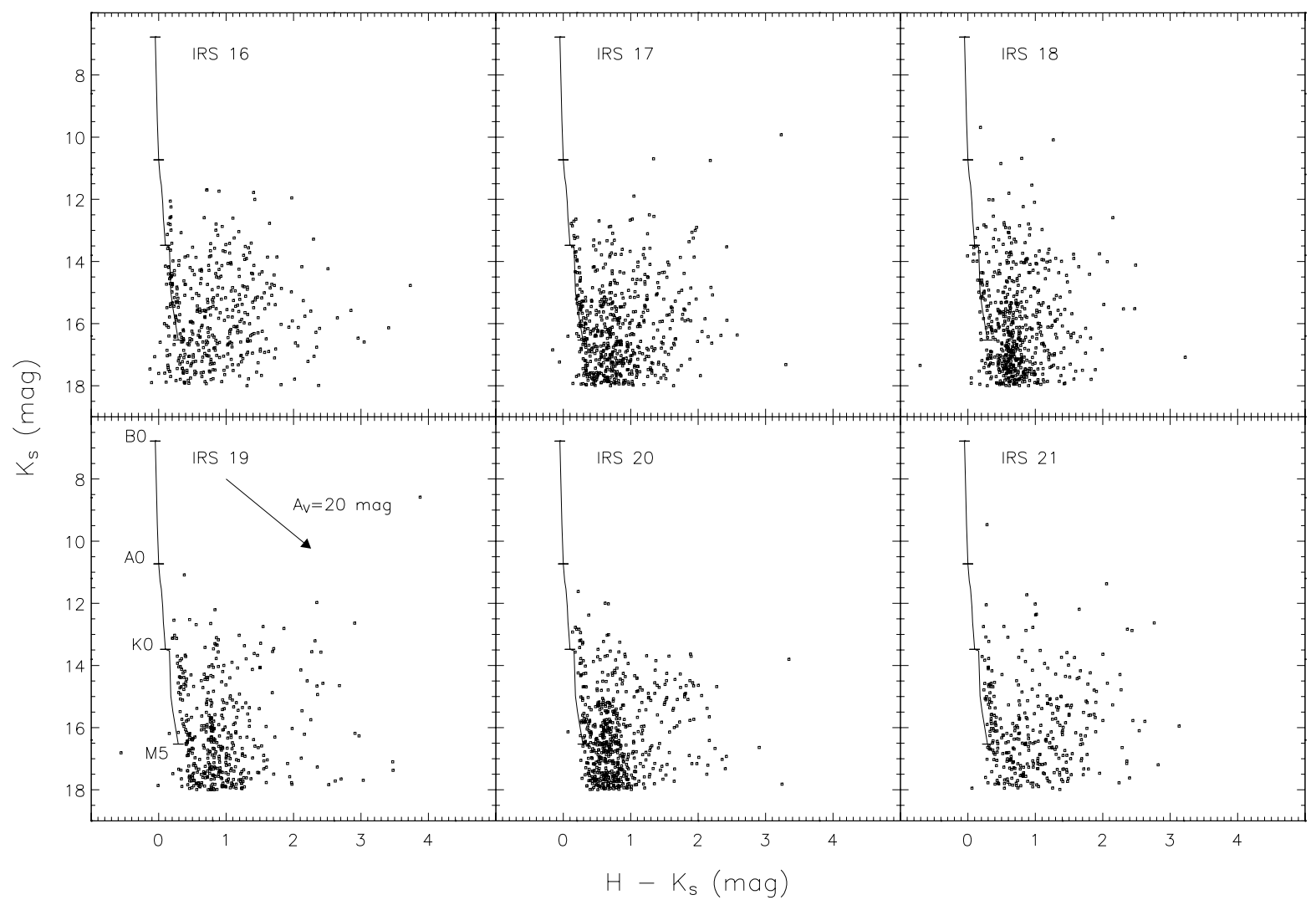

Fig. 6. Colour-magnitude diagrams (i.e., $K_{\mathrm{s}}$ vs. $H-K_{\mathrm{s}}$ ) of the 6 fields, for objects lying within 2 arcmin from the clusters' centres and up to $K_{\mathrm{s}}=18$. The solid line is the Zero Age Main Sequence (from Allen 1976 and Koornneef 1983) at the distance of the VMR, whereas the arrow is for a reddening $A_{\mathrm{V}}=20$ mag (Rieke \& Lebofsky 1985). The location of B0, A0, K0 and M5 ZAMS stars is labelled.

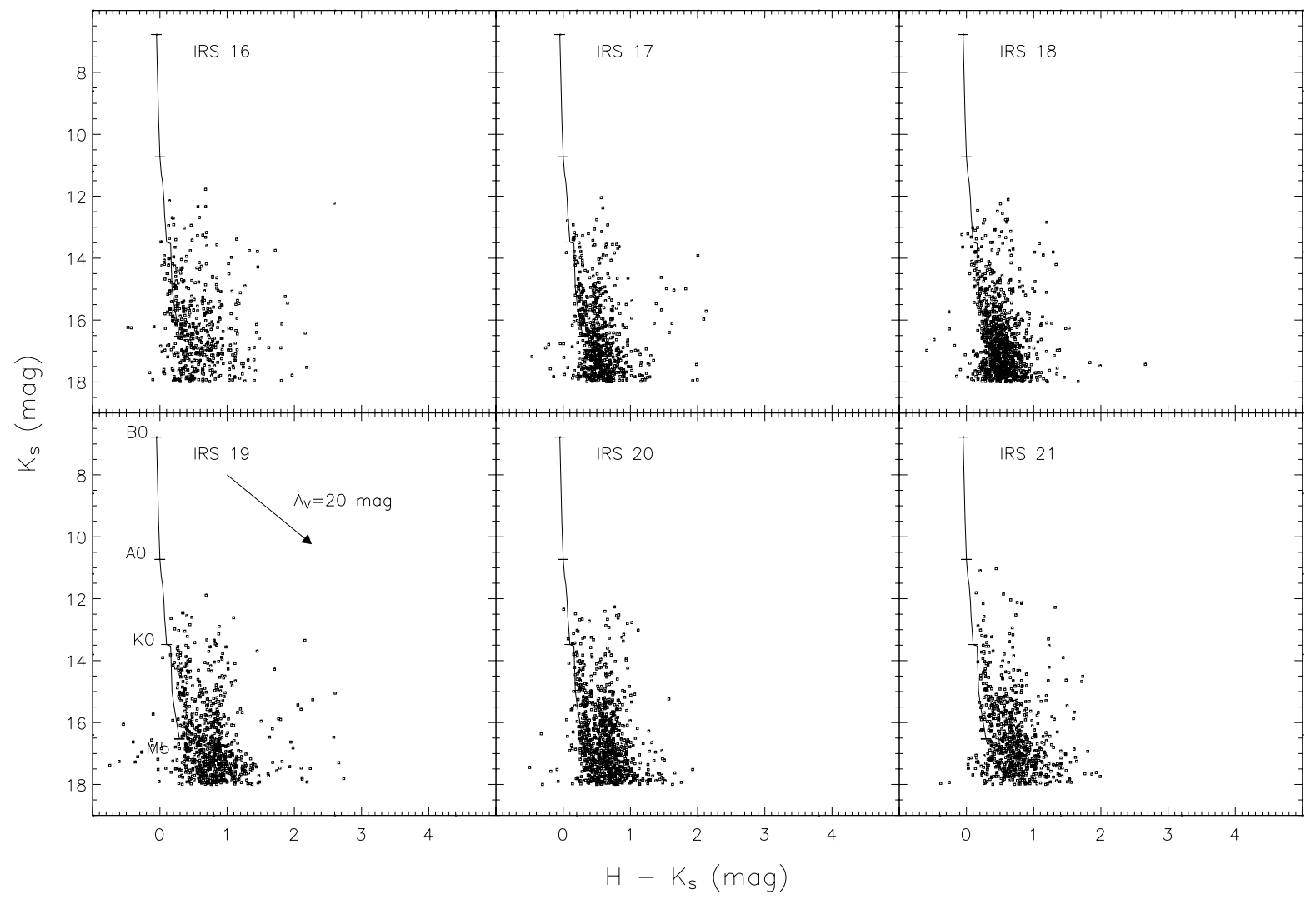

Fig. 7. Same as Fig 6, but for objects more than 2 arcmin from the clusters' centres. 


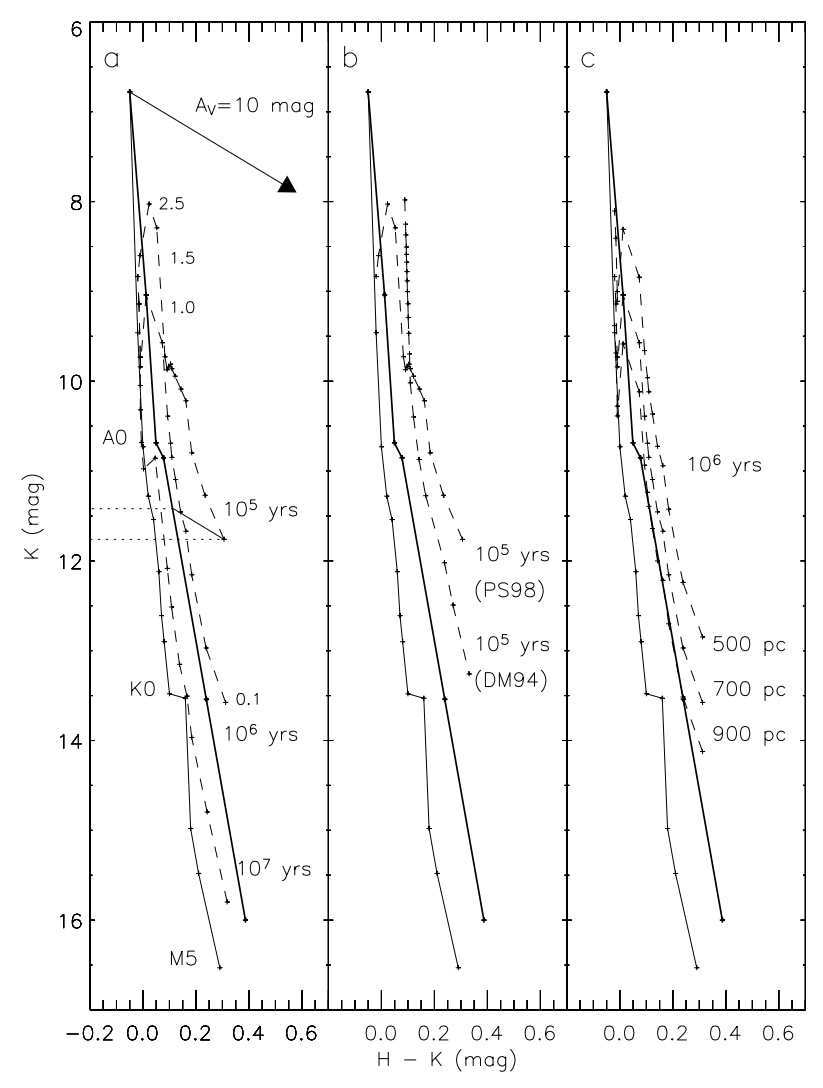

Fig. 8. a) Colour-magnitude diagram showing the ZAMS locus (thin solid line), three isochrones $\left(10^{5}, 10^{6}\right.$ and $\left.10^{7} \mathrm{yrs}\right)$ from the pms tracks by Palla \& Stahler (1999; dashed lines) and a "mean" locus used to deredden NIR sources (thick solid line), all for a distance of $700 \mathrm{pc}$. A few spectral types on the ZAMS and star masses on the $10^{6} \mathrm{yrs}$ isochrone are labelled, along with the ages of isochrones. An arrow shows the reddening for $A_{\mathrm{V}}=10$ mag according to Rieke \& Lebofsky (1985). The error made in dereddening a $0.1-M_{\odot} 10^{5}$-yrs-old pms star is indicated by the horizontal short-dashed lines. b) same as a), but only the ZAMS locus, the $10^{5}$ yrs isochrones from Palla \& Stahler (1999; labelled as PS98) and D'Antona \& Mazzitelli (1994; labelled as DM94) and the mean locus are drawn. c) same as a), but only the ZAMS locus, the $10^{6}$ yrs isochrone from Palla \& Stahler (at a distance of 500, 700 and $900 \mathrm{pc}$, labelled) and the mean locus are drawn.

than $10^{6} \mathrm{yrs}$, the error in $K$ due to the procedure is $\lesssim 0.2 \mathrm{mag}$, less than the adopted KLF binning interval $(0.5 \mathrm{mag})$. Figure $8 \mathrm{a}$ shows the worst case, one of a $0.1-M_{\odot} 10^{5}$-yrs-old pms star, whose obtained $K$ is 0.28 mag less than its actual $K$ after dereddening to the mean locus. Hence, only if a large fraction of cluster members were very young low-mass stars, the dereddened KLF could be significantly deformed. Even so, Fig. 8b shows that this result depends on the adopted evolutionary tracks: using those of D'Antona \& Mazzitelli (1994) somewhat reduces the discrepancy. Finally, Fig. 8c shows that an error on the cluster distance cannot cause a significant deformation of the dereddened KLF with respect to the actual "unreddened" one.

How well the dereddening procedure works is illustrated in Fig. 9. A model "unreddened" KLF is derived from one of the statistical realizations of a cluster with the IMF of Scalo (1998), truncated at $0.1 M_{\odot}$, and using the evolutionary tracks of Palla $\&$ Stahler (1999). For each star the $J H K$ magnitudes are scaled

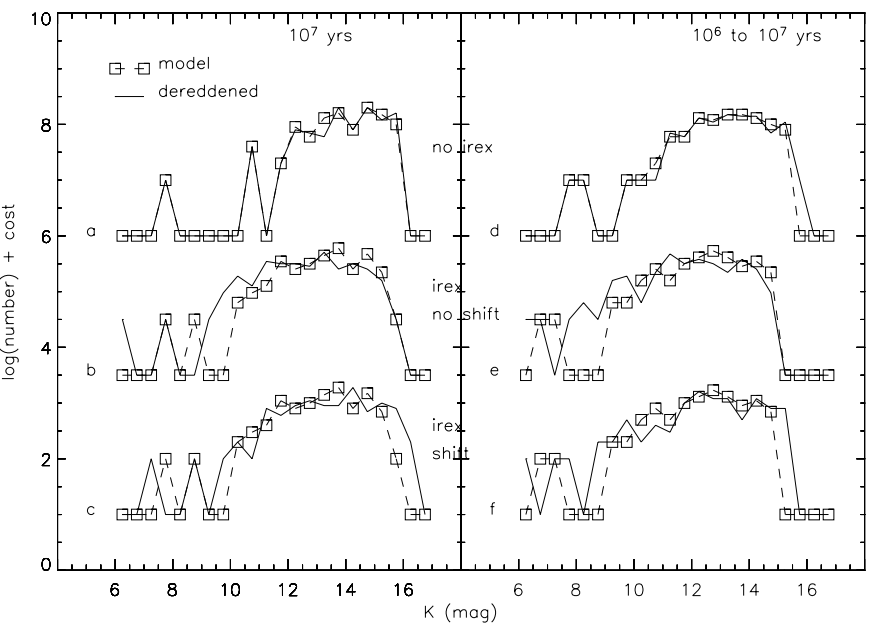

Fig. 9. Comparison of model "unreddened" KLFs (open squares, dashed line), from one of the statistical realizations of a cluster at $700 \mathrm{pc}$ as explained in the text, and "dereddened" KLFs (solid line), obtained adding a random extinction to each member of the cluster and then dereddening it according to our proposed method. Case $a$ is for a coeval $10^{7}$ years old cluster without NIR excess, case $b$ includes a NIR excess and in case $c$ the mean locus for dereddening has been shifted by 0.4 mag redward in $H-K$ in order to partially compensate for the NIR excess. Cases $d, e$ and $f$ same as $a, b$ and $c$, but for a cluster modelled with a continuous star formation from $10^{6}$ to $10^{7}$ years. Bins with a number of counts $N=0$ have been set to $\log N=-1$ and a constant equal to 7,4.5 and 2.0 has been added to $\log N$ for $a d$, be and $c f$, respectively.

to a distance of $700 \mathrm{pc}$ and reddened by adding a random value (ranging from 0 to $20 \mathrm{mag}$ ) to an overall $A_{\mathrm{V}}=20 \mathrm{mag}$ (screen), assuming the extinction law of Rieke \& Lebofsky (1985). The synthetic sample is then projected onto our adopted mean locus in the CMD and a "dereddened" KLF is constructed. Cases a and $d$ in Fig. 9 compare unreddened and dereddened KLFs for a coeval cluster of $10^{7}$ years old and for one with continuous star formation from $10^{6}$ to $10^{7}$ years old, respectively, when no NIR excess is superposed to the photospheric emission. It is evident that the "model" and the "dereddened" KLFs are almost identical.

Next, we tested how much the dereddened KLFs are affected by the NIR infrared excess of the clusters' members. Thus, we added to each star of the synthetic sample a random $K$ contribution modelled as in Hillenbrand \& Carpenter (2000). As shown in Fig. 9 for cases $b$ and e (same as a and d but with a NIR excess), the high luminosity end of the "dereddened" distribution stretches with respect to the "unreddened" KLF. A slightly better matching is obtained by shifting the mean locus in $H-K$ redward of 0.4 mag (roughly half the maximum $H-K$ excess found by Hillenbrand \& Carpenter 2000), as illustrated by cases $\mathrm{c}$ and $\mathrm{f}$.

The effect of changing the distance of the synthetic cluster or the evolutionary tracks is shown in Fig. 10. The cluster of case a above (coeval star formation, $10^{7}$ yrs old) was again synthesized but using the tracks of D'Antona \& Mazzitelli (1994), both without (case a) and with (case d) NIR excess; it is evident that the choice of the evolutionary tracks underlying the modelled cluster does not affect much the dereddened KLF which 


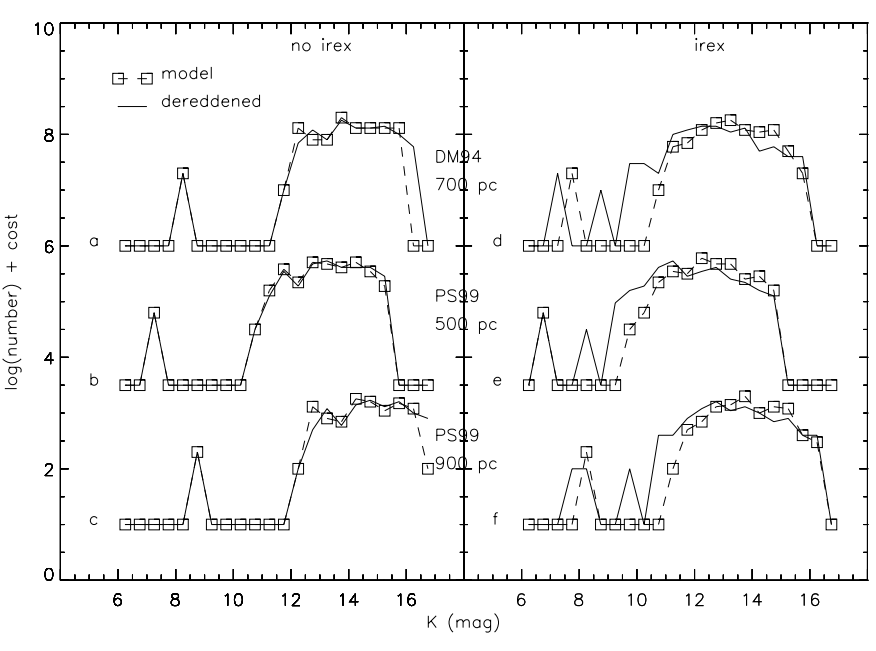

Fig. 10. Comparison of model "unreddened" KLFs (open squares, dashed line), for the same cluster (of $10^{7} \mathrm{yrs}$ ) as case $a$ of Fig. 9, and "dereddened" KLFs (solid line), obtained adding a random extinction to each member of the cluster and then dereddening it according to our proposed method. Cases $a$ and $d$ are for the evolutionary tracks of D'Antona \& Mazzitelli (1994) without $(a)$ and with $(d)$ a random NIR excess. In cases $b$ (without NIR excess) and $e$ (with NIR excess) the cluster has been put to a distance of $500 \mathrm{pc}$, whereas in cases $c$ (without NIR excess) and $f$ (with NIR excess) it has been put to a distance of $900 \mathrm{pc}$. Bins with a number of counts $N=0$ have been set to $\log N=-1$ and a constant equal to 7.0, 4.5 and 2.0 has been added to $\log N$ for $a d$, be and $c f$, respectively.

is obtained, whereas the effect of the NIR excess is the same as in Fig. 9.

The mean locus has been derived "theoretically" by assuming a distance of $700 \mathrm{pc}$, so we tested also what could happen if the actual clusters were at a different distance. Thus the cluster of case a in Fig. 9 (coeval star formation, $10^{7}$ yrs old, tracks of Palla \& Stahler 1999) was put at different distances. In Fig. 10 the dereddened KLF is shown for a distance of $500 \mathrm{pc}$ (b without NIR excess, e with NIR excess) and 900 pc (c without NIR excess, f with NIR excess). Clearly, the "dereddened" KLF always reproduces quite well the "unreddened" KLF when no NIR excess is included. When a random NIR excess is added, the deformation of the "dereddened" KLF at the high luminosity end does appear similar for all cluster models illustrated in Figs. 9 and 10.

We repeated this kind of visual test for a number of different synthesized clusters, not shown here, varying the number of members, the age and the star formation history. We found that when no NIR excess is included the "dereddened" KLF always matches quite well the "model" one for coeval clusters of $10^{6} \mathrm{yrs}$ old, whereas behaves slightly worse for $10^{8} \mathrm{yrs}$ old clusters. Anyway, the surveyed clusters are very unlikely to be so old. The "dereddened" KLF for extremely young clusters $\left(\lesssim 10^{5}\right.$ yrs old) should appear narrower than the actual one, mimicking an even younger age; we did not perform experiments on such an unevolved star population, since probably the obtained KLF is much more sensitive to the adopted evolutionary model (see, e.g., Fig. 8).

We carried out also another simple test in order to get an unbiased assessment of the goodness of the dereddening technique. We constructed 6 synthetic coeval clusters of age ranging from $10^{5.5}$ to $10^{8}$ years in steps of 0.5 (in logarithm). From these, we got the actual "unreddened" KLFs with and without a random NIR excess component. In both cases, a variable reddening was added to each synthetic member and the KLFs were then dereddened, using both the original mean lous and the one shifted in $H-K$ by 0.4 mag to compensate for the NIR excess. Each of the "dereddened" KLFs was compared to all the "unreddened" ones and the mean squared differences $\left(\chi^{2}\right)$ in counts per magnitude bin (of width $0.5 \mathrm{mag}$ ) were computed. The results can be summarized as follows:

1. when no NIR excess is included, the least $\chi^{2}$ is obtained for the same age;

2. when a NIR excess component is included, the best fit is generally obtained for the same age, but an ambiguity arises between the $10^{6.5} \mathrm{yrs}$ old and the $10^{7} \mathrm{yrs}$ old models. The same occurs when shifting the mean locus in $H-K$, although the obtained $\chi^{2}$ values are lower than before;

3. using the evolutionary tracks of D'Antona \& Mazzitelli (1994) in synthesizing the clusters, the best fits are always obtained for the same age, excepted that the dereddened KLF for a $10^{8}$ yrs old cluster (including a NIR excess component) is equally well fitted by unreddened KLFs $10^{7.5}$ and $10^{8}$ yrs old;

4. when the cluster distance is set to $500 \mathrm{pc}$, the best fits are for the same age, but when NIR excess is included, the $10^{6}$ and $10^{7}$ yrs old "dereddened" KLFs look younger by 0.5 (in logarithm). Shifting the mean locus in $H-K$ corrects for the effect, but causes the $10^{7.5}$ yrs old "dereddened" KLF to be fitted by the $10^{8}$ yrs old KLF;

5. when the cluster distance is set to $900 \mathrm{pc}$, the best fits are always for the same age, excepted that shifting the mean locus in $H-K$ causes the $10^{6.5}$ yrs old "dereddened" KLF to be well fitted by the $10^{7}$ yrs old KLF.

We performed similar tests by constructing 3 new synthetic clusters with continuous star formation in the ranges $10^{5.5}-10^{6.5}, 10^{6.5}-10^{7.5}$ and $10^{7.5}-10^{8.5}$ yrs. Now, the "dereddened" KLF is always best fitted by the "unreddened" KLF for the same cluster age range, unrespective of the presence of a random NIR excess. Comparing the "dereddened" coeval KLFs to the 3 unreddened ones with continuous star formation, indicates that only for the oldest coeval clusters some ambiguity may arise with KLFs of evolved cluster with continuous star formation. In conclusion, our dereddening technique allows to reconstruct the actual KLF with sufficient accuracy provided that the adopted mass-luminosity relation and distance to the VMR are within reasonable values and that no NIR excess is included. When the latter is not negligible, the dereddening technique stretches the "dereddened" KLF at the high luminosity end with respect to the "unreddened" one, but the age of the cluster appears still discernible within a factor of $10^{0.5}$.

By dereddening the KLFs of the observed clusters as explained, we found only small differences between the dereddened KLFs either using the mean locus or the shifted one. So, at last we decided to deredden the clusters' KLFs onto the unshifted mean locus. This also because, as can be seen in Fig. 4, only a fraction of sources exhibit a NIR excess, whereas our 


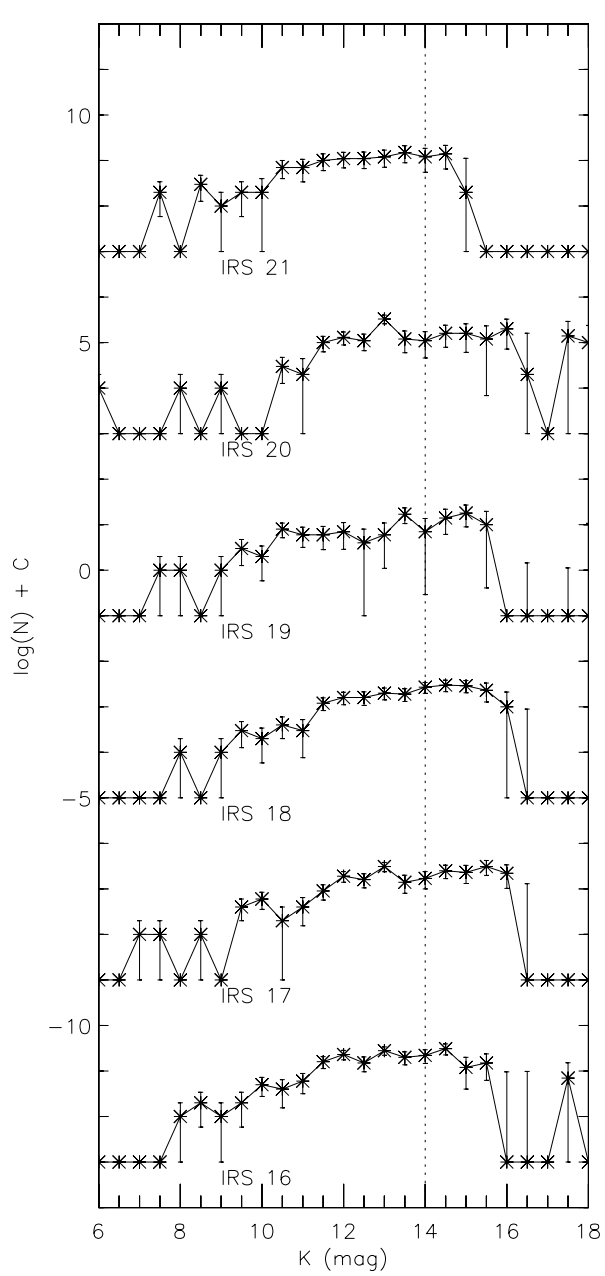

Fig. 11. The KLFs of the 6 clusters, corrected for the field stars contribution and dereddened according to the method described in the text. The vertical dotted line marks the estimated completeness limit.

tests always assumed that all sources had a NIR excess component. Furthermore, it is likely that the most prominent NIR excess is displayed by the brightest $K_{\mathrm{S}}$ sources, i.e., those falling in a luminosity interval where the KLFs are anyway scarcely sampled due to the poor statistic. Thus, the dereddened KLFs should be well representative of the actual unreddened ones. These are shown in Fig. 11. It is evident that all are quite similar, both in extent and in shape. An estimate of the completeness limit has been computed as follows. A vertical line is drawn enclosing the maximum $H-K$ displayed by the sources in the CMDs and its intersection is found with the line of the photometric completeness limit. Projecting this point along the reddening vector onto the mean locus then yields the "dereddened" completeness limit. This is estimated to be $K_{\mathrm{s}} \sim 14$ and it is shown in Fig. 11 as a vertical dotted line.

All KLFs appear extremely flat across the "dereddened" completeness limit, which corresponds to pms stars of $\sim 0.1 M_{\odot}$ for an age of $10^{6} \mathrm{yrs}\left(\sim 0.4 M_{\odot}\right.$ for an age of $\left.6 \times 10^{6} \mathrm{yrs}\right)$, according to the tracks of Palla \& Stahler (1999). This indicates that the underlying IMF is flat or slightly increasing across this mass. A break on the slope of the KLFs can be roughly identified at $K_{\mathrm{s}}>10$, suggesting a break in the IMFs at $<2.5 M_{\odot}$ for $10^{6} \mathrm{yrs}\left(<3.5 M_{\odot}\right.$ for $\left.6 \times 10^{6} \mathrm{yrs}\right)$. Above this mass limit,
Table 3. Significance level of the hypothesis that two cluster KLFs are the same distribution from the chi-square test. The crossing of each line and column lists the significance level for that pair of KLFs.

\begin{tabular}{lllllll}
\hline \hline $\begin{array}{l}\text { Field } \\
\text { name }\end{array}$ & IRS16 & IRS17 & IRS18 & IRS19 & IRS20 & IRS21 \\
\hline IRS16 & & 0.95 & 0.93 & 0.26 & 0.32 & 0.79 \\
IRS17 & 0.95 & & 0.61 & 0.06 & 0.52 & 0.25 \\
IRS18 & 0.93 & 0.61 & & 0.20 & 0.34 & 0.71 \\
IRS19 & 0.26 & 0.06 & 0.20 & & 0.04 & 0.96 \\
IRS20 & 0.32 & 0.52 & 0.34 & 0.04 & & 0.28 \\
IRS21 & 0.79 & 0.25 & 0.71 & 0.96 & 0.28 & \\
\hline
\end{tabular}

the source statistic is quite poor. The magnitude shifts of the KLFs with respect to each other due to small differences in the clusters' distance can be estimated as follows. The maximum projected distance between clusters is $\sim 1.5^{\circ}$, corresponding to $\sim 18 \mathrm{pc}$. Hence, the maximum distance difference along the line of sight is expected of the same order. This would cause maximum variations in the distance modulus $\sim 0.06$ mag, much less than the binning width.

The hypothesis that all KLFs arise from a same distribution can be tested now. If plausible, this would confirm that both the underlying IMF and the star formation history can have been similar for all clusters. Hence, we performed the chi-square test on all pairs of KLFs; the obtained significance levels are listed in Table 3. The KLFs are compared in the range $K_{\mathrm{s}}=10-14$; the lower limit is chosen to avoid counting problems due to saturation of the array. It can be seen that the hypothesis cannot be disproved at generally high significance levels. IRS 16, 17 and 18 exhibit quite high probabilities to represent a same distribution, whereas IRS 19 and 20 are less likely to meet the hypothesis, but still this cannot be disproved. Performing the test on the dereddened KLFs uncorrected for the field contribution generally causes slightly higher levels for the larger significance cases and lower levels for the smaller significance cases, but of the same order as before. In summary, the probability that the observed KLFs are drawn from a same distribution appears significant.

\subsection{Deriving the initial mass function of the clusters}

As a last step, we want to derive the IMF underlying the clusters' KLFs. So far, most of the efforts in the literature have been devoted towards constructing synthetic KLFs from a given IMF and star formation history (see, e.g., Lada \& Lada 2003). These are then fitted to the observed KLFs and the IMF underlying the best-fit KLF is thus extracted. Due to the limited statistic of our clusters, we chose to directly obtain the IMF by using the relation:

$\frac{\mathrm{d} N}{\mathrm{~d} \log M}=\frac{\mathrm{d} N}{\mathrm{~d} K}\left(\frac{\mathrm{d} \log M}{\mathrm{~d} K}\right)^{-1}$.

Note that this IMF is still model-dependent since the massmagnitude relation has to be derived from a set of evolutionary tracks. And a star formation history has to be assumed.

We computed the mass-magnitude relation from the evolutionary tracks of Palla \& Stahler (1999) for a grid of ages 


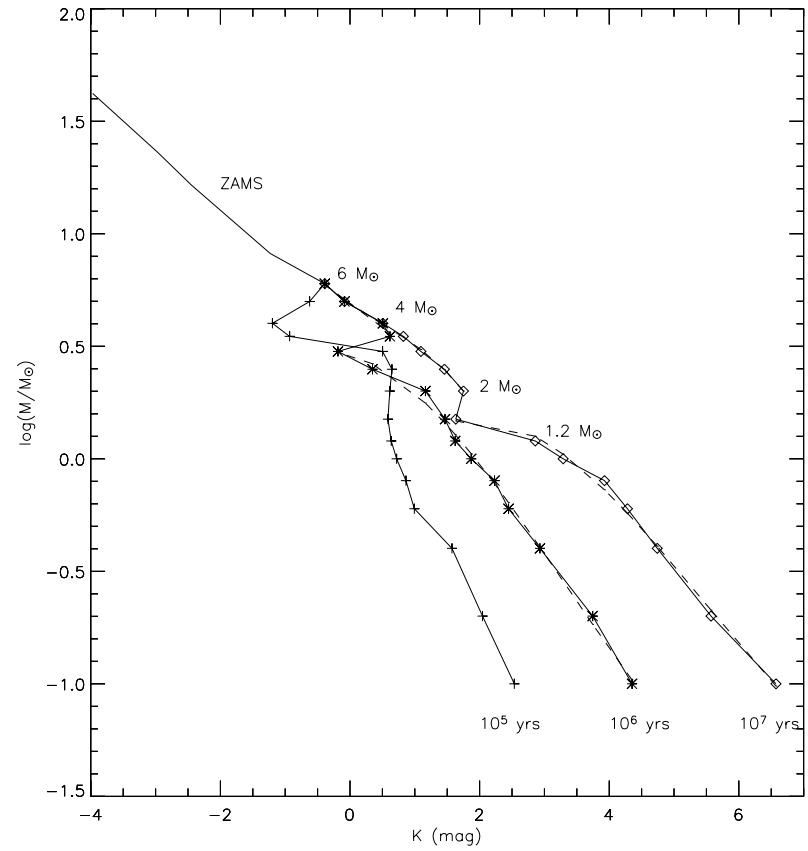

Fig. 12. Mass-magnitude relation for pms stars $10^{5}$ (plusses), $10^{6}$ (asterisks) and $10^{7}$ (open diamonds) yrs old, from the evolutionary tracks of Palla \& Stahler (1999). Masses range from 6 (upper end) to 0.1 (lower end) $M_{\odot}$ (a few points are indicated). Fits to the tracks are shown as dashed lines. The mass-magnitude relation for ZAMS stars is also drawn $\left(M>6 M_{\odot}\right)$

spanning $10^{5}$ to $10^{7} \mathrm{yrs}$. If the clusters were coeval, Eq. (1) could be applied straightforwardly and the IMF could be obtained directly from the KLF. This because the freezing of time allows one to fix the mass-magnitude relation and a given magnitude corresponds uniquely to a given mass (excepted in small magnitude intervals; see below). However, in order to apply Eq. (1) to the clusters' KLFs, both the KLFs and the derivative of the mass-magnitude relation have also to change slowly. Whereas this is true for the KLFs, it can be seen in Fig. 12 that there are limited intervals in magnitude where this condition is not satisfied by the derivative of the mass-magnitude relation. In this respect, there appear to be two kinds of problems. The first one is evident for very young ages (see the track $10^{5} \mathrm{yrs}$ old), where $\mathrm{d} \log M / \mathrm{d} K \rightarrow \infty$ around $1.5 M_{\odot}$. This is a signature of the birthline (see, e.g., Fig. 10 of Palla \& Stahler 1993) and, hence, reflects the initial conditions of the pms contraction. The other problem is common to all ages and is represented by a sort of "tooth" moving from higher to lower masses at increasing ages. This causes a small magnitude interval to be "degenerate" in mass (i. e., stars with different masses display the same $K$ ). This appears to be related to the end of the radiative contraction and the start of the main sequence. We avoided an interval spanning 1 magnitude around the points where $\mathrm{d} \log M / \mathrm{d} K \rightarrow \infty$ and the whole range (increased by $0.25 \mathrm{mag}$ at each end) where the masses are "degenerate". Outside these intervals, we used polynomial fits to approximate the mass-magnitude relation (see Fig. 12). In turn, these have been used in order to convert $K$ into masses and to estimate $\mathrm{d} \log M / \mathrm{d} K$.
As for massive stars, according to the Palla \& Stahler scenario an object enters the ZAMS after accreting 8-10 $M_{\odot}$. Hence, we used the mass-luminosity relation as derived from the ZAMS for stars with $M>6 M_{\odot}$. We adopted the absolute magnitudes given by Panagia (1973) and the colours by Koornneef (1983) to obtain the $K$ magnitudes of OB stars. The masses were then determined by converting the bolometric luminosities given by Panagia (1973) according to the formulae by Tout et al. (1996). This is also shown in Fig. 12. For an assessment of the errors affecting this relation see, e.g., Vacca et al. (1996).

The clusters' IMFs for assumed ages of $10^{6}, 3 \times 10^{6}$ and $6 \times$ $10^{6}$ yrs and coeval star formation, are shown in Fig. 13. These are compared with the IMFs from Scalo (1998) and Kroupa et al. (1993). The Scalo's IMF (in the form $\mathrm{d} N / \mathrm{d} \log M$ ) is approximated by a power law with $\Gamma=-1.7$ between $10-1 M_{\odot}$ and $\Gamma=-0.2$ between $1-0.1 M_{\odot}$. It is derived by a compilation of different determinations. That of Kroupa et al. (1993) is representative of field stars and is similar to the Scalo's (1998). In particular, $\Gamma=-1.7$ for $M>1 M_{\odot}, \Gamma=-1.2$ between $1-0.5 M_{\odot}$ and $\Gamma=-0.3$ between $0.5-0.08 M_{\odot}$.

The errors, propagated from the statistical uncertainties of the KLFs, are drawn only for $10^{6}$ yrs old. Because of the intervals to be avoided, the IMF $10^{6}$ yrs old is not defined for $M>1.6 M_{\odot}$ whereas the one $3 \times 10^{6}$ yrs old exhibits a gap between 1.6 and $4 M_{\odot}$. Moreover, at this stage we do not show the IMF for the most massive stars through the ZAMS massluminosity relation because of the poor statistic. However, it is clear that the clusters' IMFs are consistent with the "comparison" ones within the uncertainties. In the case of IRS 19, the shape of the IMF seems to be affected by the poor statistic rather than by actual differences with the comparison IMFs.

In a few cases, a better agreement would be obtained by shifting the break point envisaged in the Scalo (1998) IMF from 1 to $\sim 1.5 M_{\odot}$, especially if older ages are assumed. But it is difficult to assess whether this reflects an actual difference. We also checked that using the evolutionary tracks of D'Antona \& Mazzitelli (1994) does not affect significantly the obtained IMFs, excepted for $10^{5}$ yrs.

Assuming a coeval star formation is obviously an oversimplification. Nevertheless, if star formation is peaked at a given age, this should not change the results. Furthermore, it is evident from Fig. 12 that the derivative of the mass-magnitude relation changes slowly with time between $1-2$ and $0.1 M_{\odot}$. What changes is the $K$ magnitude. We can hope that the results should be still little affected even in the case of continuous star formation if it lasts for a time such that the change in $K$ is $\lesssim 0.5 \mathrm{mag}$ (i.e., the binning interval of our KLFs). This probably translates, e.g., into a duration of $\$ 2 \times 10^{6}$ yrs for ages of a few $10^{6}$ yrs. Some concerns arose from the possibility that star formation may span $\sim 10^{7}$ yrs and accelerate, as proposed by Palla \& Stahler (1999). Hence, we approximated clusters of 100-200 members with accelerated star formation by adding a KLF synthesized in the coeval $10^{6}$ yrs old case to a KLF synthesized in the case of continuum star formation from $10^{7}$ to $10^{6} \mathrm{yrs}$, with different fractions of the total member number. Then, we derived the IMF from the cumulative KLF by using our method. We checked that the ob- 


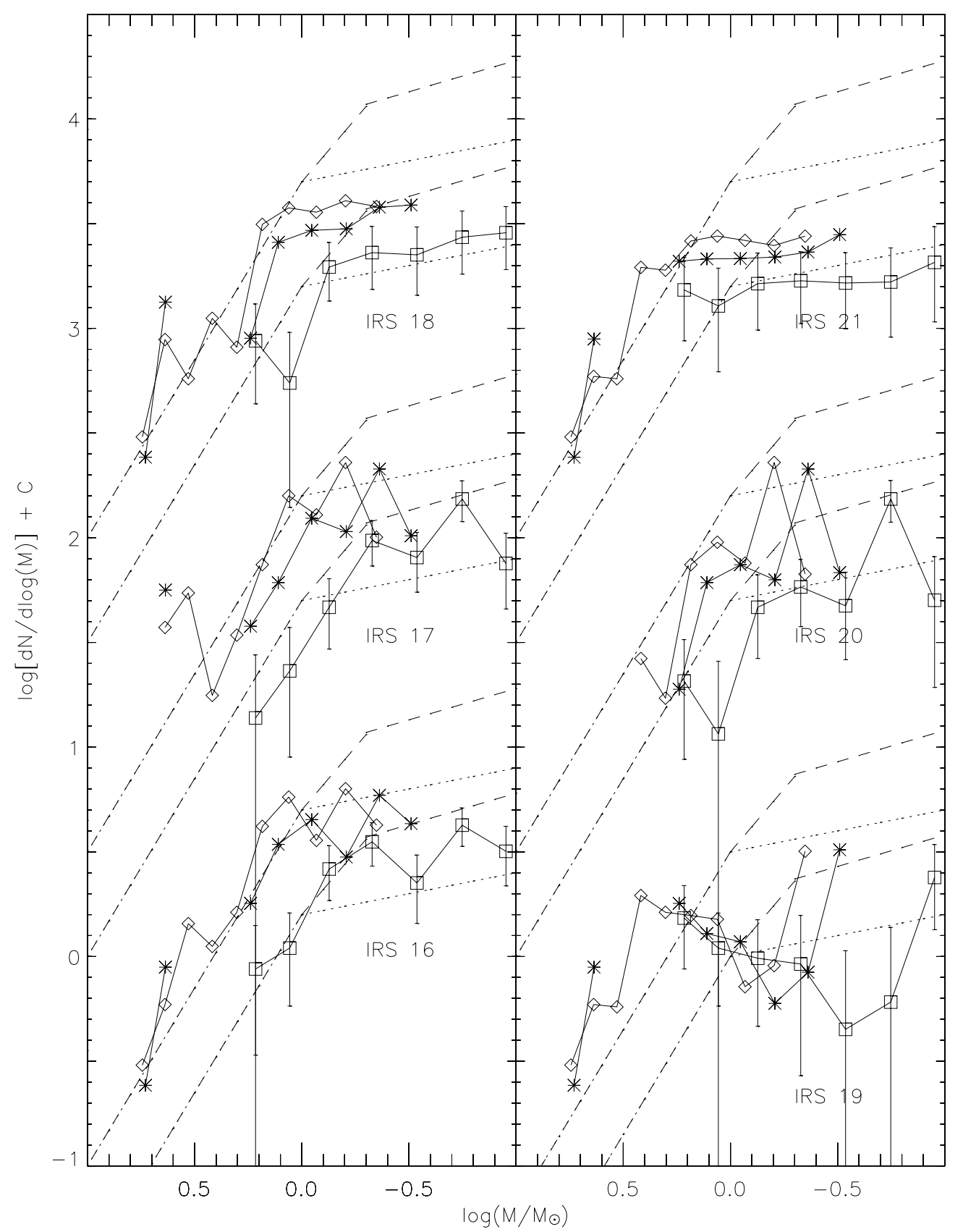

Fig. 13. The Initial Mass Functions for the 6 clusters, derived assuming coeval star formation and an age of $10^{6}$ (open squares), $3 \times 10^{6}$ (asterisks) and $6 \times 10^{6}$ (open diamonds) yrs. All cluster IMFs are compared with the IMFs from Scalo (1998; dotted line) and Kroupa et al. (1993; dashed lines), each with two different normalizations. For the sake of clarity, errorbars are drawn only for $10^{6}$ yrs old.

tained IMF is very similar to the underlying one (Scalo-like) in the $1.5-0.1 M_{\odot}$ range even if up to a $35 \%$ of the synthesized population is produced through continuum star formation. In particular, in a $\log (\mathrm{d} N / \mathrm{d} \log M)$ vs. $\log M$ plot the purely coeval IMF and the mixed one still coincide within the statistical errors. This may be understood analytically: the continuum component of the KLF is flatter and spread on a larger magnitude interval than the coeval component, and its underlying area is also much smaller. But we checked that even increasing the continuum fraction of the synthesized population to a $50 \%$, the obtained IMF does not change dramatically, although appearing slightly flatter. However, this assumes that the underlying IMF is the same in the two population components; only in the unlikely case that stars of different masses are originated at very different times we could expect major differences between the obtained and the actual IMF in the $1.5-0.1 M_{\odot}$ range.

We could reverse our point by trying to constrain the clusters' age assuming that the underlying IMF is a standard one, like our comparison IMFs. If so, the clusters' KLFs are consistent with coeval star formation and an age between 1-6 $\times$ $10^{6}$ yrs. Younger ages can be discarded since they produce IMFs peaked at $0.3-0.1 M_{\odot}$ with Salpeter-like slopes. Also, clusters older than $10^{7}$ yrs can be discarded, since the resulting IMFs would be peaked at $>2 M_{\odot}$ and declining towards higher and smaller masses. 
Table 4. Clusters' properties derived from their IMF. Column 2 lists the number of cluster members (down to the mass completeness limit), Col. 3 the cluster total mass (as in Col. 2), Col. 4 the mass of the most massive star within the cluster and Col. 5 the star formation efficiency.

\begin{tabular}{ccccc}
\hline \hline $\begin{array}{c}\text { Field } \\
\text { name }\end{array}$ & Members & Mass & $\begin{array}{c}\text { Largest } \\
\text { star } \\
\left(M_{\odot}\right)\end{array}$ & SFE \\
\hline IRS 16 & $144 \pm 15$ & 103 & 7.1 & $0.24^{a}$ \\
IRS 17 & $126 \pm 15$ & 90 & 6.5 & $0.26^{b}$ \\
IRS 18 & $124 \pm 14$ & 89 & 6.5 & - \\
IRS 19 & $69 \pm 13$ & 49.5 & 4.4 & $0.095^{b}$ \\
& & & & $0.21^{a}$ \\
IRS 20 & $98 \pm 14$ & 70 & 5.5 & $0.8^{b}$ \\
IRS 21 & $95 \pm 14$ & 68 & 5.4 & $0.11^{a}$ \\
\hline
\end{tabular}

${ }^{a}$ Gas mass from Yamaguchi et al. (1999).

${ }^{b}$ Gas mass from Wouterloot \& Brand (1999).

\section{Discussion}

Knowing the dereddened cluster KLFs allows us to estimate the number of members $N_{\mathrm{T}}$ up to the completeness magnitude (i.e., down to $0.1-0.4 M_{\odot}$ ), to be compared with $I_{\mathrm{c}}$ in Table 2 . The results are listed in Table 4 and it can be noted that $N_{\mathrm{T}}$ is roughly twice the corresponding value of $I_{\mathrm{c}}$. Clearly, the latter underestimates the true number of members, probably due to the fact that the sky contribution is estimated in a region still contaminated by stars from the cluster population. In fact, deriving the clusters' KLFs within a radius of 1 arcmin (instead of 2 arcmin) results in a much lower $N_{\mathrm{T}}$.

If we want to include in $N_{\mathrm{T}}$ all clusters' members down to the hydrogen burning limit, then the values derived from our observations have to be corrected for the age. Having proved that the clusters' IMF is very similar to standard ones, like those of Scalo (1998) or Kroupa et al. (1993), by adopting for it the IMF of Scalo (1998) we can estimate the correction to apply to $N_{\mathrm{T}}$. E.g., for a $6 \times 10^{6} \mathrm{yrs}$ old cluster, our completeness magnitude corresponds to a mass limit of $0.4 M_{\odot}$. Integrating the Scalo IMF from 0.4 to $0.1 M_{\odot}$ yields $\sim 55 \%$ of stars in that mass range, so the observed $N_{\mathrm{T}}$ should be increased by a factor of 2.2. This largely exceeds the statistical (counting) uncertainties listed in Table 2 . Note that a further underestimate is likely to arise from having selected sky areas close to the clusters in order to construct the control KLFs (i.e., possibly still containing a small number of clusters' members). And we do not consider here the brown dwarf population (which is not sampled by our observation).

Actually, $N_{\mathrm{T}}$ is bound to be underestimated anyway, due to the unknown fraction of close companions which escape observations. How critical this problem may be is demonstrated by Fig. 6 of Giannini et al. (2005); using ISAAC images of IRS 17, these authors can resolve source \# 40 of Massi et al. (1999) into 6 different components. We checked that the same star in our $K_{\mathrm{s}}$ image is resolved into only 3 components, although the brightest ones.

The total mass (down to the hydrogen burning limit) of the clusters can be derived, as well, by integrating the Scalo IMF times the mass. The results are listed in Table 4 and, like $N_{\mathrm{T}}$, can be underestimated by a factor 2.2 or so, depending on the age. The highest expected stellar mass $x$ in each cluster was estimated by integrating the Scalo IMF, as well, from $\infty$ to a mass $x$ and requiring that the result is 1 . The obtained values (see Table 4) are consistent with the bolometric luminosities given by Massi et al. $(2000,2003)$ and confirm that the most massive stars in the 6 clusters are intermediate-mass stars. Note that an increase in $N_{\mathrm{T}}$ of a factor 2.2 would roughly double $x$.

At last, we can derive the star formation efficiencies of the parental clumps if the mass of the associated gas is known. We used the mm $\left(\mathrm{C}^{18} \mathrm{O}\right)$ observations of Wouterloot \& Brand (1999) and Yamaguchi et al. (1999). Because of the low resolution of the latter data, these probably refer to matter on a larger scale than found by Wouterloot \& Brand (1999), i.e. clouds rather than clumps or cores, and are likely to underestimate the gas content of these larger scale structures. Nevertheless, all derived efficiencies are $\leq 26 \%$, excepted towards IRS 20 where a star formation efficiency of $\sim 80 \%$ is found. These are typical values for young embedded clusters (Lada \& Lada 2003). Instead, if taken at face value, the high star formation efficiency towards IRS 20 implies that the cluster is emerging as a bound structure (Lada \& Lada 2003); however, we remark that the area around IRS 20 covered by the mm observations of Wouterloot \& Brand (1999) is very limited and the gas mass could then have been underestimated.

Although our NIR observations were able to clarify the shape of the clusters' IMF between 1-3 and 0.1-0.4 $M_{\odot}$, they fail to strongly constrain the clusters' age. This would have allowed to more accurately model the dereddened KLFs and derive the underlying IMF. Also, the substellar mass regime is not probed by our observations, requiring much deeper imaging. However, as shown in Fig. 11, the dereddened KLFs maintain roughly the same slope above the completeness magnitude (remember that 5 fields are $\sim 1$ mag deeper than the 6th one). No strong decrease is evidenced; hence, because of uncompleteness, the KLFs can at most increase above this limit. This means either that the clusters are slightly older than $1 \times 10^{6}$ yrs and stars fainter than $K_{\mathrm{s}}=14$ are still above the hydrogen burning limit (and follow a Scalo-like IMF), or that the clusters are as old as, or younger than, $1 \times 10^{6} \mathrm{yrs}$ and the IMF itself is still increasing at the hydrogen burning limit (assumed $\mathrm{d} \log M / \mathrm{d} K$ does not exhibit major changes below $0.1 M_{\odot}$ ).

As for the high mass end of the IMF, this is not only affected by the poor statistic. The brightest sources in our $J H K_{\mathrm{s}}$ images are also saturated and, probably, most of the brighter $K_{\mathrm{s}}$ sources are largely affected by a NIR excess emission. However, the data obtained by Massi et al. $(1999,2003)$ are less affected by saturation or non-linearity problems. As for IRS 16, it coincides with an HII region and these authors found that it is excited by a B0-B5 source, which is the most massive cluster's member (consistently with the highest expected stellar mass listed in Table 4, given also the uncertainty on $N_{\mathrm{T}}$ ). In the case of the remaining clusters, the most massive clusters' members are the sources emerging in the NIR and identified by Massi et al. (1999) as the counterparts of the IRAS point sources. Whereas the clusters' IMF is consistent with the mass of the most massive star in each cluster, imaging surveys of IRAS 


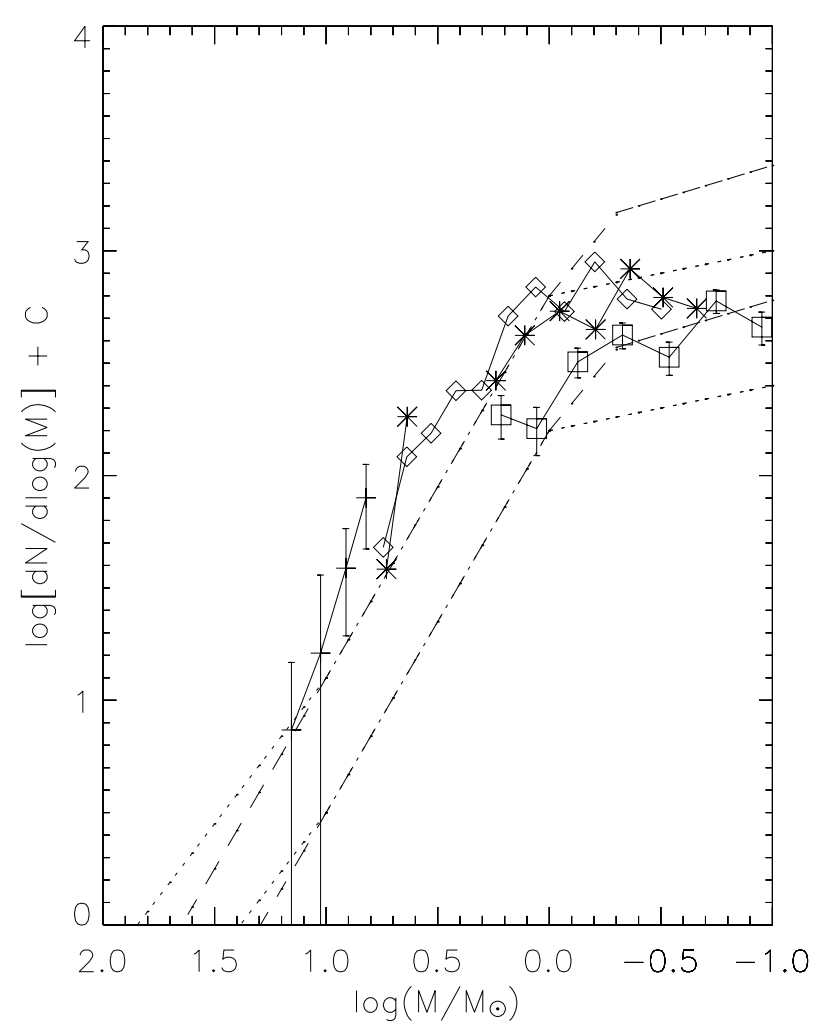

Fig. 14. Sum of the 6 initial mass functions, same symbols as Fig. 13. The four most massive bins have been derived using the ZAMS massmagnitude relation discussed in the text.

point sources in the VMR have so far failed to unveil high luminosity IRAS sources associated with isolated young stars (Massi et al. 1999, 2003).

A way to investigate the high mass end of the clusters' IMF is by adding together all 6 IMFs, as to increase the statistic. This should also approximate the field star IMF, if most stars originate in clusters and then disperse. The "global" IMF is shown in Fig. 14. The four most massive bins have been obtained by using the ZAMS mass-magnitude relation discussed in Sect. 4.7. We dropped three points with resulting masses $>20 M_{\odot}$. We checked that these are due to the young stars or protostars lying in the centre of IRS 17, IRS 19 and IRS 20 and identified by Massi et al. (1999), then members of the clusters. But, as shown by Massi et al. (2000, 2003), based on the measured bolometric luminosity and the upper limit on the radio continuum emission, they are intermediate-mass, not high-mass (proto-)stars. Clearly, they exhibit a strong NIR excess emission and the ZAMS mass-magnitude relation cannot be applied to them.

The global IMF is quite similar to the comparison ones, particularly at $>10^{6} \mathrm{yrs}$. The IMF $10^{6}$ yrs old implies a break at $M>1 M_{\odot}$, but the mass of many objects in the intermediatemass regime may have been overestimated because of the NIR excess. At the high-mass end, the IMF slope appears slightly steeper than that of the comparison ones, but here the statistic is still poor. However, it is noteworthy a drop-off of the IMF at $M \sim 10 M_{\odot}$. There are 13 stars in the 4 most massive bins $\left(M \sim 6 M_{\odot}\right)$, of which 1 in the most massive one $\left(M \sim 14 M_{\odot}\right)$ and 2 in the next most massive one $\left(M \sim 10 M_{\odot}\right)$.
This indicates that none of the 6 clusters could produce a highmass stars, although they all originated intermediate-mass stars as predicted, e. g., by the Scalo's IMF based on their number of members. But applying the same statistical argument, since we are now sampling a total number of stars $>656$ (see Table 4) we should find a star with $M>22.5 M_{\odot}$ (i.e., an O8-09 ZAMS star). Such an object would have a bolometric luminosity $>10^{4} L_{\odot}$ and form an HII region. There is not such a massive source within the 6 clusters (Massi et al. 2000, 2003). Hence, the drop-off appears to be a real feature of the global IMF.

If the actual clusters' IMF is a standard one, then the most massive stars in each cluster depend on the cluster mass, as expected based on statistical grounds. But they fail to follow statistic when added together, suggesting that the dependence on the cluster mass is "physical". I.e., high-mass stars need large clusters to be formed. On the other hand, it may be that the IMF in the whole region is a standard one, while that of the single clusters is much steeper at the high mass end. Then, we should find massive stars elsewhere in the region. Massi et al. (1999, 2003) do not find massive stars in their D-cloud sample of IRAS sources, but those objects might well have escaped the selection criteria adopted by Liseau et al. (1992). This would reconcile with a statistical view of the IMF, but if the clusters' IMF is steeper than that of field stars or open clusters, it is likely so because of different physical conditions in the parental gas, so, in a sense, still "physical". A careful multi-wavelength examination of the whole region is then necessary in order to settle this point.

\section{Conclusions}

We have presented the results of a Near-Infrared photometric survey of a sample of 6 embedded young clusters, all associated with luminous IRAS sources in the Vela D molecular cloud. They lie at the same distance and have been imaged with the same instrumental setting; this allowed us to derive the clusters' properties in a consistent way, without most of the observational biases often affecting the comparison of different star forming regions. The clusters' $K$ Luminosity Functions have been obtained from the same images, by subtracting a control $K$ Luminosity Function, for sources $>2$ ' from the clusters' centre, from that for sources $<2^{\prime}$ from the clusters' centre. A simple method for dereddening the $K$ Luminosity Functions, based on colour-magnitude diagrams, has been developed and described. The main results are the following:

1. Our images confirm the previous results of Massi et al. (2000, 2003), i.e., all selected fields contain young embedded clusters with a radius $\sim 0.1-0.2 \mathrm{pc}$ and a mean stellar

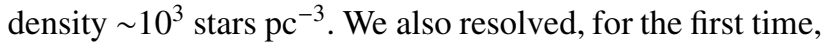
IRS 17 into 2 sub-clusters.

2. The dereddened clusters' $K$ Luminosity Functions have been compared in pairs using the chi-square test; the hypothesis that all arise from a same distribution cannot be disproved at significance levels ranging from 0.04 to 0.95 (but $>0.50$ in most cases). This suggests that the underlying 
Initial Mass Function and star formation history may have been quite similar.

3. The clusters' Initial Mass Functions, obtained from the dereddened $K$ Luminosity Functions in the hypothesis of coeval star formation, are compatible with standard ones, like those of Scalo (1998) and Kroupa et al. (1993), for assumed ages ranging from $10^{6}$ to $6 \times 10^{6} \mathrm{yrs}$. Major departures would occur if the clusters were either younger or older than assumed. However, they are unlikely to be older than $5 \times 10^{6}$ yrs since all of them are still associated with molecular gas.

4. The global IMF, obtained by adding together all 6 single IMFs, is similar to a standard IMF. But it appears steeper at the high-mass end and exhibits a fall off at $\sim 10 M_{\odot}$. E.g., on statistical grounds the Scalo IMF would predict a star of $>22.5 M_{\odot}$ within one of the cluster, which is not found. Then, either high-mass stars need larger clusters to be formed, or the single IMFs are steeper at the high-mass end because of the physical conditions in the parental gas.

5. Based on their $K$ Luminosity Functions, the clusters' members range from 69 (IRS 19) to 144 (IRS 16). Assuming an Initial Mass Function like Scalo (1998) yields total clusters' masses ranging from 50 to $100 M_{\odot}$. If the clusters are $10^{6} \mathrm{yrs}$ old, the stellar mass is sampled down to $0.1 M_{\odot}$. If the clusters are as old as $6 \times 10^{6}$ yrs old, both number of members and mass have to be increased by a factor $\sim 2.2$ to reach the same mass lower limit.

6. The star formation efficiency ranges from 0.1 to 0.8 , being $<0.26$ in all cases but one (IRS 20).

Acknowledgements. We thank the ESO staff for the excellent support during the observations at the NTT. We also thank the referee, Bruce Elmegreen, whose comments greatly improved the quality of this work. FM acknowledges partial support from "fondi finalizzati INAF, bando 2002: Disks and Jets in High-mass Star Forming Regions".

\section{References}

Adams, F. C., \& Myers, P. 2001, ApJ, 533, 744

Allen, C. W. 1976, Astrophysical Quantities (3rd ed.) (London: Athlone press)

Baraffe, I., Chabrier, G., Allard, F., \& Hauschildt, P. H. 1998, A\&A, 337,403

Bonnell, I. A., \& Clarke, C. J. 1999, MNRAS, 309, 461

Clarke, C. J., Bonnell, I. A., \& Hillenbrand, L. A. 2000, The Formation of Stellar Clusters, in Protostars and Planets IV, ed. V. Mannings, A. Boss, \& S. S. Russell (Tucson: University of Arizona press), 151
D’Antona, F., \& Mazzitelli, I. 1994, ApJS, 90, 467

de Wit, W. J., Testi, L., Palla, F., Vanzi, L., \& Zinnecker, H. 2004, A\&A, 425, 937

de Wit, W. J., Testi, L., Palla, F., \& Zinnecker, H. 2005, A\&A, 437, 247

Giannini, T., Massi, F., Podio, L., et al. 2005, A\&A, 433, 941

Gomez, M., Hartmann, L., Kenyon, S. J., \& Hewett, R. 1993, AJ, 105, 1927

Koornneef, J. 1983, A\&A, 128, 84

Kroupa, P., Tout, C. A., \& Gilmore, G. 1993, MNRAS, 262, 545

Hillenbrand, L. A., \& Carpenter, J. M. 2000, ApJ, 540, 236

Hillenbrand, L. A., \& Hartmann, L. W. 1998, ApJ, 492, 540

Lada, C. J., \& Lada, E. A. 2003, ARA\&A, 41, 57

Liseau, R., Lorenzetti, D., Nisini, B., Spinoglio, L., \& Moneti, A. 1992, A\&A, 265, 577

Lorenzetti, D., Spinoglio, L., \& Liseau, R. 1993, A\&A, 275, 489

Massi, F., Giannini, T., Lorenzetti, D., et al. 1999, A\&AS, 136, 471

Massi, F., Lorenzetti, D., Giannini, T., \& Vitali, F. 2000, A\&A, 353, 598

Massi, F., Lorenzetti, D., \& Giannini, T. 2003, A\&A, 399, 147

Massi, F., Elia, D., Giannini, T., Lorenzetti, D., \& Nisini, B. 2005, A Large Scale View of Molecular Gas and Dust Towards Cloud D of the Vela Molecular Ridge, in Proc. of the Dusty and Molecular Universe: a Prelude to Herschel and ALMA, ed. A. Wilson (ESA SP-577), 389

Muench, A. A., Lada, E. A., \& Lada, C. J. 2000, ApJ, 533, 358

Murphy, D. C., \& May, J. 1991, A\&A, 247, 202

Palla, F., \& Stahler, S. W. 1993, ApJ, 418, 414

Palla, F., \& Stahler, S. W. 1999, ApJ, 525, 772

Panagia, N. 1973, AJ, 78, 929

Persson, S. E., Murphy, D. C., Krzeminski, W., Roth, M., \& Rieke, M. J. 1998, AJ, 116, 2475

Scalo, J. 1998, The IMF Revisited: A Case for Variations, in The Stellar Initial Mass Function, Proc. of the 38th Herstmonceux Conf., ed. G. Gilmore, \& D. Howell, ASP Conf. Ser., 142, 201

Rieke, G. H., \& Lebofsky, M. J. 1985, ApJ, 288, 618

Testi, L., Palla, F., Prusti, T., Natta, A., \& Maltagliati, S. 1997, A\&A, 320, 159

Testi, L., Palla, F., \& Natta, A. 1998, A\&AS, 133, 81

Testi, L., Palla, F., \& Natta, A. 1999, A\&A, 342, 515

Testi, L., Palla, F., \& Natta, A. 2001a, The Onset of Cluster Formation around Intermediate-Mass Stars, in From Darkness to Light: Origin and Evolution of Young Stellar Clusters, ed. T. Montmerle, \& Ph. André, ASP Conf. Ser., 243, 377

Testi, L., Vanzi, L., \& Massi, F. 2001b, ESO Mess., 103, 28

Tout, C. A., Pols, O. R., Eggleton, P. P., \& Han, Z. 1996, MNRAS, 281, 257

Vacca, W. D., Garmany, C. D., \& Shull, J. M. 1996, ApJ, 460, 914

Zinnecker, H., McCaughrean, M. J., \& Wilking, B. A. 1993, The Initial Stellar Population, in Protostars and Planets III, ed. E. H. Levy, \& J. I. Lunine (Tucson: The University of Arizona Press), 429

Wouterloot, J. G. A., \& Brand, J. 1999, A\&AS, 140, 177

Yamaguchi, N., Mizuno, N., Saito, H., et al. 1999, PASJ, 51, 775 